\title{
Distribution analysis of the fire severity characteristics of single passenger road vehicles using heat release rate data
}

\author{
Mohd Zahirasri Mohd Tohir ${ }^{*}$ and Michael Spearpoint
}

\begin{abstract}
Fires associated with vehicles have the potential to impact on life safety and property protection. The fire severity characteristics of single passenger vehicle fires are presented in this paper by the total energy released, peak rate of heat release and the time to peak rate of heat release using experimental data collated from the literature. Risk-based fire design can be supported by data presented in a statistical form such that passenger vehicles are categorized by their curb weight and probability distribution curves are obtained for each fire severity characteristic. Analysis of the data shows that the total energy released and the time to peak rate of heat release are generally shown to exhibit an increasing trend with curb weight.
\end{abstract}

Keywords: Passenger vehicle; Heat release rate; Fire severity; Probability distribution

\section{Introduction \\ Background}

Fires in vehicles can impact on the life safety of the vehicle occupants and the people in the vicinity of the fire. Vehicle fires can also result in material losses both in terms of the vehicle itself but also to neighbouring property. Therefore it is prudent to understand the risks of vehicle fires and the need to potentially reduce their probability of occurring and/or mitigate the severity if a fire does occur. This review is part of a larger research investigation into risk-based fire safety of passenger road vehicles in parking buildings being undertaken at the University of Canterbury.

Heat release rate is an important fire severity characteristic relating to fire safety (Babrauskas and Peacock 1992). The heat release rate time-history defines the growth of the fire and other related characteristics such as the peak heat release rate, the time to the peak and the total energy release which in turn determine some measures of the severity of the fire particularly when considering the impacts to people and property remote from the vehicle itself. Moreover, the fire environment can be assessed using the heat release rate information

\footnotetext{
* Correspondence: mzm10@uclive.ac.nz

Department of Civil and Natural Resources Engineering, University of Canterbury, Private Bag 4800 Christchurch 8140, New Zealand
}

as input to a calculation. For example, the smoke layer height as a measure of tenability can be predicted by a computational model once the heat release rate is determined. In addition, the tenability components of smoke such as obscuration, toxic species and heat may need to be determined and these components can often be obtained as a function of the heat release rate. Thus, this review compiles the available heat release rates curves for a single passenger road vehicles from the current literature to form a resource for calculations such as the fire spread between vehicles, fire and smoke conditions in enclosures (such as car parks or tunnels), etc.

With the increased consideration of performancebased fire safety analysis in New Zealand and elsewhere, the design fire concept is a critical component for the design of buildings (Fleischmann 2008). There is developing interest in using probabilistic assessment methods as part of a risk-based approach to performance-based fire safety design. These methods provide an objective quantification of risk which could lead to an optimization of the selection of fire protection measures in a cost-effective manner. Hence, there is a need to compile data in a statistical form and this review introduces a set of probability distributions for the heat release rate characteristics of passenger road vehicles. These distributions provide researchers and designers with a starting point

\section{Springer}


for risk-based design of parking buildings or other similar structures that involve vehicles.

\section{Vehicle classification}

The definition of passenger vehicle used in this review is based on the New Zealand Transport Authority (NZTA) which states that it is a motor vehicle constructed primarily for the carriage of passengers, with not more than nine seating positions which include the driver's seating position, and either has at least four wheels or it has three wheels and a gross vehicle mass exceeding one tonne (NZTA 2012). Therefore, the passenger vehicle data collected here are limited by this definition, which excludes other ground-based passenger vehicles such as buses, trains etc.

However even within the NZTA definition of a passenger vehicle there is likely to be a wide range of vehicle sizes and types so it is useful to consider classifying vehicles into smaller groups. There are numerous of ways to categorize passenger vehicles and different regulations and jurisdictions have a variety of definitions for the purposes of classification. Some of the most common classifications are the vehicle engine size, the vehicle dimensions (e.g. length, interior volume size), the vehicle seating capacity, the vehicle curb weight, age, or wheelbase (Opland 2007). For this review the American National Standards Institute (ANSI) (American National Standard 2007) classification system based on the curb weight of the vehicle is adopted (Table 1) as the mass is identified in this paper as a key parameter related to the potential fire load of vehicles. For example, previous work by (Joyeux et al. 2002) provides total energy release values for five car categories such that the higher the average mass of the vehicles the higher the total energy released. Similarly research by (Shintani et al. 2004) shows an increasing linear trend for the correlation of body weight against total energy released from four vehicle fire experiments. As such, it seems reasonable to investigate further how the heavier the vehicle the more total energy that will likely be released during the fire. It is also useful to consider how the peak heat release rate

Table 1 ANSI classification of vehicles by curb weight

\begin{tabular}{lc}
\hline Classification & Curb weight \\
\hline Passenger car: Mini & $1500-1999 \mathrm{lbs}(680-906 \mathrm{~kg})$ \\
Passenger car: Light & $2000-2499 \mathrm{lbs}(907-1134 \mathrm{~kg})$ \\
Passenger car: Compact & $2500-2999 \mathrm{lbs}(1135-1360 \mathrm{~kg})$ \\
Passenger car: Medium & $3000-3499 \mathrm{lbs}(1361-1587 \mathrm{~kg})$ \\
Passenger car: Heavy & $\geq 3500 \mathrm{lbs}(\geq 1588 \mathrm{~kg})$ \\
Van/MPV & Not defined \\
SUV & Not defined \\
\hline
\end{tabular}

Table 2 Percentage of passenger vehicle population classified by weight in some European countries

\begin{tabular}{lcccc}
\hline & $<\mathbf{1 0 0 0} \mathbf{~ k g}$ & $\begin{array}{c}\mathbf{1 0 0 0}- \\
\mathbf{1 2 4 9} \mathbf{~ g g}\end{array}$ & $\begin{array}{c}\mathbf{1 2 5 0}- \\
\mathbf{1 4 9 9} \mathbf{~ k g}\end{array}$ & $\geq \mathbf{1 5 0 0} \mathbf{~ g g}$ \\
\hline Netherlands & 33.7 & 31.0 & 24.8 & 10.4 \\
Estonia & 8.1 & 31.5 & 32.3 & 28.1 \\
Spain & 22.6 & 34.1 & 31.8 & 11.5 \\
Finland & 11.7 & 28.9 & 36.4 & 23.1 \\
Cyprus & 27.4 & 33.6 & 23.8 & 15.1 \\
Latvia & 7.4 & 30.7 & 32.9 & 29.1 \\
Norway & 10.1 & 27.6 & 36.2 & 26.1 \\
Switzerland & 8.6 & 23.5 & 30.6 & 37.4 \\
Poland & 33.3 & 31.0 & 20.0 & 15.7 \\
Portugal & 0.6 & 5.7 & 28.0 & 65.7 \\
Average, \% & $\mathbf{1 6 . 3}$ & $\mathbf{2 7 . 8}$ & $\mathbf{2 9 . 7}$ & $\mathbf{2 6 . 2}$ \\
\hline
\end{tabular}

and the time to peak heat release rate for vehicles changes as the vehicle mass increases.

To further argue for the classification of passenger vehicle by mass, data from the EU (2012) shown in Table 2 illustrates how vehicles are divided into bands determined on mass and also shows that the population of vehicles varies between different countries. Therefore a risk-based assessment on vehicle fires might need to account for this variation particularly if it is accepted that the vehicle mass relates to the severity of a vehicle fire.

However, this selection of classification has its own weaknesses considering that different weight classes do not necessarily directly relate to the amount of combustible material in a vehicle. Based on the report by (Swift 2012), the usage of plastics/composites in light vehicles has been increasing steadily from 1960s up to 2010 s. Thus, the age of the vehicles is also an important factor which may affect the severity of the fire and this is further investigated below.

Table 3 Number of experiments by ANSI classification

\begin{tabular}{lc}
\hline ANSI classification & No. of experiments \\
\hline Passenger car: Mini & 6 \\
Passenger car: Light & 7 \\
Passenger car: Compact & 7 \\
Passenger car: Medium & 5 \\
Passenger car: Heavy & 7 \\
Van/MPV & 6 \\
SUV & 2 \\
Unclassified vehicle & 1 \\
\hline
\end{tabular}


Table 4 Passenger car: mini

\begin{tabular}{|c|c|c|c|c|c|c|c|c|c|c|c|c|c|}
\hline ID & $\begin{array}{l}\text { Vehicle make } \\
\text { and model }\end{array}$ & Vehicle year & $\begin{array}{l}\text { Curb } \\
\text { weight } \\
\text { (kg) }\end{array}$ & $\begin{array}{l}\text { Facility } \\
\text { type }\end{array}$ & Condition & $\begin{array}{l}\text { Ignition } \\
\text { source }\end{array}$ & $\begin{array}{l}\text { Ignition } \\
\text { location }\end{array}$ & $\begin{array}{l}\text { Incipient } \\
\text { stage }\end{array}$ & $\begin{array}{l}\text { Mass loss } \\
\text { rate }\end{array}$ & $\begin{array}{l}\text { Toxic product } \\
\text { emission/Product } \\
\text { consumption }\end{array}$ & $\begin{array}{c}\text { Heat release } \\
\text { rate evaluation } \\
\text { method }\end{array}$ & $\begin{array}{c}\text { Smoke } \\
\text { production }\end{array}$ & $\begin{array}{l}\text { Reference and experiment } \\
\text { date (ED)/report submitted } \\
\text { (RS)/date published (DP) }\end{array}$ \\
\hline \multirow[t]{2}{*}{ M1 } & \multirow{2}{*}{$\begin{array}{l}\text { Trabant } \\
\text { Limousine }\end{array}$} & \multirow{2}{*}{$\begin{array}{l}\text { Undetermined, } \\
\text { available from } \\
1963-1990\end{array}$} & \multirow[t]{2}{*}{695} & \multirow{2}{*}{$\begin{array}{c}\text { Room } \\
\text { calorimeter }\end{array}$} & \multirow{2}{*}{$\begin{array}{l}\text { Slight gap } \\
\text { at top of } \\
\text { windows }\end{array}$} & \multirow{2}{*}{$\begin{array}{c}250 \mathrm{~mL} \\
\text { isopropanol }\end{array}$} & \multirow{2}{*}{$\begin{array}{l}\text { Front } \\
\text { seat }\end{array}$} & \multirow[t]{2}{*}{ Unclear } & \multirow[t]{2}{*}{$\mathrm{N}$} & \multirow[t]{2}{*}{$\mathrm{N}$} & \multirow{2}{*}{$\begin{array}{l}\text { Convective } \\
\text { calorimetry }\end{array}$} & \multirow[t]{2}{*}{$\mathrm{N}$} & (Steinert 2000) \\
\hline & & & & & & & & & & & & & ED 1998 \\
\hline \multirow[t]{2}{*}{ M2 } & \multirow[t]{2}{*}{ Renault 5} & \multirow[t]{2}{*}{ 1980s } & \multirow[t]{2}{*}{757} & \multirow{2}{*}{$\begin{array}{c}\text { Corner } \\
\text { calorimeter }\end{array}$} & \multirow[t]{2}{*}{$\mathrm{N} / \mathrm{A}$} & \multirow{2}{*}{$\begin{array}{l}1.5 \mathrm{~L} \\
\text { gasoline in } \\
\text { open tray }\end{array}$} & \multirow{2}{*}{$\begin{array}{l}\text { Under } \\
\text { left front } \\
\text { seat }\end{array}$} & \multirow{2}{*}{$\begin{array}{l}\text { Not } \\
\text { mentioned }\end{array}$} & \multirow[t]{2}{*}{ Y } & \multirow[t]{2}{*}{$\mathrm{N}$} & \multirow{2}{*}{$\begin{array}{l}\text { Species-based } \\
\text { calorimetry }\end{array}$} & \multirow[t]{2}{*}{ N } & (Joyeux 1997) \\
\hline & & & & & & & & & & & & & ED 24 Jul 1995 \\
\hline \multirow[t]{2}{*}{ M3 } & \multirow[t]{2}{*}{ Unknown } & \multirow[t]{2}{*}{1995} & \multirow[t]{2}{*}{830} & \multirow{2}{*}{$\begin{array}{c}\text { Open } \\
\text { calorimeter }\end{array}$} & \multirow[t]{2}{*}{ N/A } & \multirow{2}{*}{$\begin{array}{l}1 \mathrm{~L} \\
\text { gasoline in } \\
\text { open tray }\end{array}$} & \multirow{2}{*}{$\begin{array}{l}\text { Under } \\
\text { gear } \\
\text { box }\end{array}$} & \multirow{2}{*}{$\begin{array}{c}\text { Not } \\
\text { mentioned }\end{array}$} & \multirow[t]{2}{*}{ Y } & $\mathrm{N}$ & Species based & N & (Joyeux 1997) \\
\hline & & & & & & & & & & & & & ED 5 Jul 1996 \\
\hline M4 & Rover-Austin & $1990 \mathrm{~s}$ & 893 & Room & Slight gap & $250 \mathrm{~mL}$ & Front & Unclear & $\mathrm{N}$ & $\mathrm{N}$ & Convective & N & (Steinert 2000) \\
\hline & Metro LS & & & calorimeter & $\begin{array}{l}\text { at top of } \\
\text { windows }\end{array}$ & isopropanol & & & & & calorimetry & & ED 1998 \\
\hline M5 & Opel Kadett & Undetermined, & 737- & Parking & N/A & N/A & N/A & Unclear & Y & $\mathrm{N}$ & Mass loss & $N$ & (Van Oerle et al. 1999) \\
\hline & & $\begin{array}{l}\text { available from } \\
1962-1991\end{array}$ & 1007 & garage & & & & & & & & & DP 5 Nov 1999 \\
\hline M6 & Fiat 127 & Undetermined, & $705-870$ & Road & N/A & N/A & N/A & Unclear & $N$ & $\mathrm{~N}$ & Not mentioned & $\mathrm{N}$ & (Carvel 2004) \\
\hline & & $\begin{array}{l}\text { available from } \\
1971-1983\end{array}$ & & & & & & & & & & & ED 1997 \\
\hline
\end{tabular}


Table 5 Passenger car : light

\begin{tabular}{|c|c|c|c|c|c|c|c|c|c|c|c|c|c|}
\hline ID & $\begin{array}{l}\text { Vehicle } \\
\text { make } \\
\text { and } \\
\text { model }\end{array}$ & Vehicle year & $\begin{array}{l}\text { Curb } \\
\text { weight } \\
\text { (kg) }\end{array}$ & $\begin{array}{l}\text { Facility } \\
\text { type }\end{array}$ & Condition & $\begin{array}{l}\text { Ignition } \\
\text { source }\end{array}$ & $\begin{array}{l}\text { Ignition } \\
\text { location }\end{array}$ & $\begin{array}{l}\text { Incipient } \\
\text { stage }\end{array}$ & $\begin{array}{l}\text { Mass } \\
\text { loss } \\
\text { rate }\end{array}$ & $\begin{array}{c}\text { Carbon } \\
\text { emission/ } \\
\text { Product } \\
\text { consumption }\end{array}$ & $\begin{array}{l}\text { Heat } \\
\text { release } \\
\text { rate } \\
\text { evaluation } \\
\text { method }\end{array}$ & $\begin{array}{c}\text { Smoke } \\
\text { production }\end{array}$ & $\begin{array}{c}\text { Reference and } \\
\text { experiment date } \\
(E D) / \text { report } \\
\text { submitted (RS)/date } \\
\text { published (DP) }\end{array}$ \\
\hline \multirow[t]{2}{*}{ L1 } & \multirow{2}{*}{$\begin{array}{l}\text { Datsun } \\
160 \mathrm{~J} \\
\text { Sedan }\end{array}$} & \multirow[t]{2}{*}{ Late 1970s } & \multirow[t]{2}{*}{918} & \multirow[t]{2}{*}{$\begin{array}{l}\text { Open } \\
\text { calorimeter }\end{array}$} & \multirow{2}{*}{$\begin{array}{c}\text { All doors closed, left front } \\
\text { window completely open, } \\
\text { other windows rolled } \\
\text { down } 5 \mathrm{~cm}\end{array}$} & \multirow{2}{*}{$\begin{array}{l}3 \mathrm{~L} \text { of } \\
\text { heptane in } \\
\text { open tray }\end{array}$} & \multirow[t]{2}{*}{$\begin{array}{l}\text { Under the } \\
\text { engine }\end{array}$} & \multirow[t]{2}{*}{ Included } & \multirow[t]{2}{*}{ Y } & \multirow[t]{2}{*}{$\begin{array}{l}\mathrm{Y}\left(\mathrm{CO} \& \mathrm{CO}_{2}\right. \\
\text { production) }\end{array}$} & \multirow[t]{2}{*}{$\begin{array}{l}\text { Oxygen } \\
\text { depletion }\end{array}$} & \multirow[t]{2}{*}{ Y } & $\begin{array}{l}\text { (Mangs and Keski- } \\
\text { Rahkonen 1994) }\end{array}$ \\
\hline & & & & & & & & & & & & & RS 6 May 1993 \\
\hline L2 & $\begin{array}{l}\text { Ford } \\
\text { Taurus }\end{array}$ & Late 1970s & 990 & $\begin{array}{c}\text { Open } \\
\text { Calorimeter }\end{array}$ & $\begin{array}{c}\text { Left door } 10 \mathrm{~cm} \text { ajar with } \\
\text { the window completely } \\
\text { open, right door closed } \\
\text { with window rolled down } \\
5 \mathrm{~cm}\end{array}$ & $\begin{array}{l}1.5 \mathrm{~L} \text { of } \\
\text { heptane in } \\
\text { open tray }\end{array}$ & $\begin{array}{l}\text { Under left } \\
\text { front seat }\end{array}$ & Included & Y & $\begin{array}{l}\mathrm{Y}\left(\mathrm{CO} \& \mathrm{CO}_{2}\right. \\
\text { production })\end{array}$ & $\begin{array}{l}\text { Oxygen } \\
\text { depletion }\end{array}$ & Y & $\begin{array}{c}\text { (Mangs and Keski- } \\
\text { Rahkonen 1994) RS } 6 \\
\text { May 1993 }\end{array}$ \\
\hline L3 & $\begin{array}{l}\text { Citroen } \\
\text { BX } 16 \text { RE }\end{array}$ & 1970 s or $1980 \mathrm{~s}$ & 1067 & $\begin{array}{l}\text { Room } \\
\text { Calorimeter }\end{array}$ & $\begin{array}{c}\text { Slight gap at top of } \\
\text { windows }\end{array}$ & $\begin{array}{c}250 \mathrm{~mL} \\
\text { isopropanol }\end{array}$ & Front seat & Unclear & N & $\mathrm{N}$ & $\begin{array}{l}\text { Convective } \\
\text { calorimetry }\end{array}$ & $N$ & $\begin{array}{c}\text { (Steinert 2000) ED } \\
1998\end{array}$ \\
\hline L4 & $\begin{array}{l}\text { Datsun } \\
180 B \\
\text { Sedan }\end{array}$ & Late 1970s & 1102 & $\begin{array}{c}\text { Open } \\
\text { Calorimeter }\end{array}$ & $\begin{array}{c}\text { All doors closed, left front } \\
\text { window completely open, } \\
\text { other windows rolled } \\
\text { down } 5 \mathrm{~cm}\end{array}$ & $\begin{array}{l}3 \mathrm{~L} \text { of } \\
\text { heptane in } \\
\text { open tray }\end{array}$ & $\begin{array}{l}\text { Under the } \\
\text { engine }\end{array}$ & Included & Y & $\begin{array}{l}\mathrm{Y}\left(\mathrm{CO} \& \mathrm{CO}_{2}\right. \\
\text { production })\end{array}$ & $\begin{array}{l}\text { Oxygen } \\
\text { depletion }\end{array}$ & Y & $\begin{array}{c}\text { (Mangs and Keski- } \\
\text { Rahkonen 1994) RS } 6 \\
\text { May } 1993\end{array}$ \\
\hline L5 & $\begin{array}{l}\text { Austin } \\
\text { Maestro }\end{array}$ & 1982 & $915-950$ & $\begin{array}{l}\text { Rail shuttle } \\
\text { car }\end{array}$ & $\begin{array}{c}\text { Driver and front passenger } \\
\text { side windows completely } \\
\text { open }\end{array}$ & $\begin{array}{l}\text { No.7 wood } \\
\text { crib (peak } \\
\text { HRR of } \\
\text { about } \\
10 \mathrm{~kW} \text { ) }\end{array}$ & On front seat & Included & $\mathrm{N}$ & $N$ & $\begin{array}{l}\text { Oxygen } \\
\text { depletion }\end{array}$ & $\mathrm{N}$ & $\begin{array}{c}\text { (Shipp and } \\
\text { Spearpoint 1995) ED } \\
18 \text { Feb } 1991\end{array}$ \\
\hline L6 & $\begin{array}{l}\text { Citroen } \\
\text { BX } 14 \text { RE }\end{array}$ & 1986 & 930 & $\begin{array}{l}\text { Rail shuttle } \\
\text { car }\end{array}$ & $\begin{array}{c}\text { Driver and front passenger } \\
\text { side windows completely } \\
\text { open }\end{array}$ & $\begin{array}{l}400 \mathrm{~mL} \\
\text { gasoline in } \\
\text { foil tray } \\
\text { (100 mL } \\
\text { spilled) }\end{array}$ & $\begin{array}{l}\text { Engine } \\
\text { compartment } \\
\text { under hood }\end{array}$ & Unclear & $\mathrm{N}$ & $\mathrm{N}$ & $\begin{array}{l}\text { Oxygen } \\
\text { depletion }\end{array}$ & $N$ & $\begin{array}{c}\text { (Shipp and } \\
\text { Spearpoint 1995) ED } \\
18 \text { Feb } 1991\end{array}$ \\
\hline L7 & $\begin{array}{l}\text { Peugeot } \\
309\end{array}$ & $\begin{array}{l}\text { Undetermined, } \\
\text { available from } \\
1985 \text { - } 1993\end{array}$ & 880-975 & $\begin{array}{l}\text { Parking } \\
\text { garage }\end{array}$ & N/A & $\mathrm{N} / \mathrm{A}$ & N/A & Unclear & Y & $\mathrm{N}$ & Mass loss & $N$ & $\begin{array}{l}\text { (Van Oerle et al. } \\
\text { 1999) DP } 5 \text { Nov } 1999\end{array}$ \\
\hline
\end{tabular}


Table 6 Passenger car : compact

\begin{tabular}{|c|c|c|c|c|c|c|c|c|c|c|c|c|c|}
\hline ID & $\begin{array}{l}\text { Vehicle } \\
\text { make } \\
\text { and } \\
\text { model }\end{array}$ & Vehicle year & $\begin{array}{c}\text { Curb } \\
\text { weight } \\
(\mathrm{kg})\end{array}$ & $\begin{array}{l}\text { Facility } \\
\text { type }\end{array}$ & Condition & $\begin{array}{l}\text { Ignition } \\
\text { source }\end{array}$ & $\begin{array}{l}\text { Ignition } \\
\text { location }\end{array}$ & $\begin{array}{l}\text { Incipient } \\
\text { stage }\end{array}$ & $\begin{array}{l}\text { Mass } \\
\text { loss } \\
\text { rate }\end{array}$ & $\begin{array}{c}\text { Carbon } \\
\text { emission/ } \\
\text { Product } \\
\text { consumption }\end{array}$ & $\begin{array}{l}\text { Heat } \\
\text { release } \\
\text { rate } \\
\text { evaluation } \\
\text { method }\end{array}$ & $\begin{array}{c}\text { Smoke } \\
\text { production }\end{array}$ & $\begin{array}{c}\text { Reference and } \\
\text { experiment date (ED)/ } \\
\text { report submitted (RS)/ } \\
\text { date published (DP) }\end{array}$ \\
\hline \multirow[t]{2}{*}{ C1 } & \multirow[t]{2}{*}{ Unknown } & \multirow{2}{*}{$\begin{array}{l}\text { Undetermined, } \\
\text { available from } \\
1970 \text { - late } \\
\text { 1990s }\end{array}$} & \multirow[t]{2}{*}{1182} & \multirow{2}{*}{$\begin{array}{c}\text { Open } \\
\text { Calorimeter }\end{array}$} & \multirow{2}{*}{$\begin{array}{l}\text { Driver and passenger } \\
\text { windows rolled down } \\
\qquad 10 \mathrm{~cm}\end{array}$} & \multirow{2}{*}{$\begin{array}{l}\text { Cloth } \\
\text { soaked } \\
\text { with } \\
\text { methanol }\end{array}$} & \multirow[t]{2}{*}{ Driver's seat } & \multirow[t]{2}{*}{ Included } & \multirow[t]{2}{*}{$\mathrm{N}$} & \multirow[t]{2}{*}{ N } & \multirow{2}{*}{$\begin{array}{l}\text { Oxygen } \\
\text { depletion }\end{array}$} & \multirow[t]{2}{*}{$\mathrm{N}$} & (Shintani et al. 2004) \\
\hline & & & & & & & & & & & & & DP March 2004 \\
\hline \multirow[t]{2}{*}{$C 2$} & \multirow[t]{2}{*}{ Unknown } & \multirow[t]{2}{*}{1995} & \multirow[t]{2}{*}{1303} & \multirow{2}{*}{$\begin{array}{c}\text { Open } \\
\text { Calorimeter }\end{array}$} & \multirow[t]{2}{*}{ N/A } & \multirow{2}{*}{$\begin{array}{l}1.5 \mathrm{~L} \\
\text { gasoline in } \\
\text { open tray }\end{array}$} & \multirow{2}{*}{$\begin{array}{l}\text { Under left } \\
\text { front seat }\end{array}$} & \multirow{2}{*}{$\begin{array}{c}\text { Not } \\
\text { mentioned }\end{array}$} & \multirow[t]{2}{*}{ Y } & \multirow[t]{2}{*}{$N$} & \multirow{2}{*}{$\begin{array}{l}\text { Species- } \\
\text { based } \\
\text { calorimetry }\end{array}$} & \multirow[t]{2}{*}{$\mathrm{N}$} & (Joyeux 1997) \\
\hline & & & & & & & & & & & & & ED 19 Jun 1996 \\
\hline \multirow[t]{2}{*}{ C3 } & \multirow[t]{2}{*}{ Unknown } & \multirow[t]{2}{*}{ 1990s } & \multirow[t]{2}{*}{1360} & \multirow{2}{*}{$\begin{array}{c}\text { Room } \\
\text { Calorimeter }\end{array}$} & \multirow{2}{*}{$\begin{array}{c}1 \mathrm{~m}^{2} \text { windows } \\
\text { opened; tank has } \\
10 \mathrm{~L} \text { of gasoline fuel. }\end{array}$} & \multirow{2}{*}{$\begin{array}{l}80 \mathrm{~g} \text { of } \\
\text { alcohol gel } \\
\text { fuel }\end{array}$} & \multirow{2}{*}{$\begin{array}{l}\text { Right rear } \\
\text { wheel }\end{array}$} & \multirow[t]{2}{*}{ Included } & $\mathrm{N}$ & $\mathrm{N}$ & Mass loss & $\mathrm{N}$ & (Okamoto et al. 2009) \\
\hline & & & & & & & & & & & & & RS 11 Sep 2007 \\
\hline C4 & Unknown & 1990s & 1360 & Room & Windows closed; tank & $80 \mathrm{~g}$ of & Right rear & Included & $\mathrm{N}$ & N & Mass loss & $\mathrm{N}$ & (Okamoto et al. 2009) \\
\hline & & & & Calorımeter & $\begin{array}{c}\text { has } 10 \text { of gasolıne } \\
\text { fuel. }\end{array}$ & $\begin{array}{l}\text { alcohol gel } \\
\text { fuel }\end{array}$ & & & & & & & RS 11 Sep 2008 \\
\hline C5 & Unknown & 1990s & 1360 & Room & Windows closed; Tank & $80 \mathrm{~g}$ of & Right rear & Included & $\mathrm{N}$ & N & Mass loss & $\mathrm{N}$ & (Okamoto et al. 2009) \\
\hline & & & & & $\begin{array}{c}\text { has } 20 \mathrm{~L} \text { of gasolıne } \\
\text { fuel. }\end{array}$ & $\begin{array}{l}\text { alcohol gel } \\
\text { fuel }\end{array}$ & & & & & & & RS 11 Sep 2009 \\
\hline C6 & Unknown & 1990s & 1360 & Room & $0.28 \mathrm{~m}^{2}$ left front & $2 \mathrm{~L}$ of & Left front & Included & $\mathrm{N}$ & $\mathrm{N}$ & Mass loss & $\mathrm{N}$ & (Okamoto et al. 2009) \\
\hline & & & & & $\begin{array}{l}\text { WIndow opened; } \\
\text { Tank has } 10 \mathrm{~L} \text { of } \\
\text { gasoline fuel. }\end{array}$ & $\begin{array}{l}\text { gasolıne } \\
\text { spilled on } \\
\text { left front } \\
\text { seat }\end{array}$ & & & & & & & RS 11 Sep 2010 \\
\hline C7 & $\begin{array}{l}\text { Ford } \\
\text { Focus }\end{array}$ & 2002 & 1197 & $\begin{array}{c}\text { Room } \\
\text { Calorimeter }\end{array}$ & $\begin{array}{c}\text { All passenger } \\
\text { windows closed, } \\
\text { bonnet closed after }\end{array}$ & $\begin{array}{l}\text { IMS soaked } \\
\text { fibre-board }\end{array}$ & $\begin{array}{c}\text { Engine } \\
\text { compartment }\end{array}$ & Included & $\mathrm{N}$ & $\mathrm{N}$ & $\begin{array}{l}\text { Species- } \\
\text { based } \\
\text { calorimetry }\end{array}$ & $\mathrm{N}$ & $\begin{array}{l}\text { (Department for } \\
\text { Communities and Local } \\
\text { Government CLG 2010) }\end{array}$ \\
\hline & & & & & fire nas established. & & & & & & & & ED 27 Aug 2008 \\
\hline
\end{tabular}


Table 7 Passenger car: medium

\begin{tabular}{|c|c|c|c|c|c|c|c|c|c|c|c|c|c|}
\hline ID & $\begin{array}{l}\text { Vehicle } \\
\text { make } \\
\text { and } \\
\text { model }\end{array}$ & Vehicle year & $\begin{array}{l}\text { Curb } \\
\text { weight } \\
\text { (kg) }\end{array}$ & $\begin{array}{l}\text { Facility } \\
\text { type }\end{array}$ & Condition & $\begin{array}{l}\text { Ignition } \\
\text { source }\end{array}$ & $\begin{array}{l}\text { Ignition } \\
\text { location }\end{array}$ & $\begin{array}{l}\text { Incipient } \\
\text { stage }\end{array}$ & $\begin{array}{l}\text { Mass } \\
\text { loss } \\
\text { rate }\end{array}$ & $\begin{array}{c}\text { Carbon } \\
\text { emission/ } \\
\text { Product } \\
\text { consumption }\end{array}$ & $\begin{array}{c}\text { Heat release } \\
\text { rate } \\
\text { evaluation } \\
\text { method }\end{array}$ & $\begin{array}{c}\text { Smoke } \\
\text { production }\end{array}$ & $\begin{array}{l}\text { Reference and experiment } \\
\text { date (ED)/report submitted } \\
\text { (RS)/date published (DP) }\end{array}$ \\
\hline \multirow[t]{2}{*}{ MED1 } & \multirow[t]{2}{*}{ Unknown } & \multirow{2}{*}{$\begin{array}{c}\text { Undetermined, } \\
\text { available from } \\
1970 \text { - late } \\
1990 \mathrm{~s}\end{array}$} & \multirow[t]{2}{*}{1380} & \multirow{2}{*}{$\begin{array}{c}\text { Open } \\
\text { Calorimeter }\end{array}$} & \multirow{2}{*}{$\begin{array}{c}\text { Driver and } \\
\text { passenger } \\
\text { windows rolled } \\
\text { down } 10 \mathrm{~cm}\end{array}$} & \multirow{2}{*}{$\begin{array}{l}\text { Cloth } \\
\text { soaked } \\
\text { with } \\
\text { methanol }\end{array}$} & \multirow{2}{*}{$\begin{array}{l}\text { Driver's } \\
\text { seat }\end{array}$} & \multirow[t]{2}{*}{ Included } & \multirow[t]{2}{*}{$\mathrm{N}$} & \multirow[t]{2}{*}{$\mathrm{N}$} & \multirow{2}{*}{$\begin{array}{l}\text { Oxygen } \\
\text { depletion }\end{array}$} & $\mathrm{N}$ & (Shintani et al. 2004) \\
\hline & & & & & & & & & & & & & DP March 2004 \\
\hline \multirow[t]{2}{*}{ MED2 } & \multirow{2}{*}{$\begin{array}{l}\text { Peugeot } \\
406 \\
\text { Berline }\end{array}$} & \multirow[t]{2}{*}{1994} & \multirow[t]{2}{*}{1382} & \multirow{2}{*}{$\begin{array}{c}\text { Corner } \\
\text { Calorimeter }\end{array}$} & \multirow[t]{2}{*}{ N/A } & \multirow{2}{*}{$\begin{array}{l}1.5 \mathrm{~L} \\
\text { gasoline } \\
\text { in open } \\
\text { tray }\end{array}$} & \multirow{2}{*}{$\begin{array}{c}\text { Under } \\
\text { gear box }\end{array}$} & \multirow[t]{2}{*}{ Included } & \multirow[t]{2}{*}{ Y } & \multirow[t]{2}{*}{$N$} & \multirow{2}{*}{$\begin{array}{l}\text { Oxygen } \\
\text { depletion }\end{array}$} & $\mathrm{N}$ & (Joyeux et al. 2002) \\
\hline & & & & & & & & & & & & & ED 1995 \\
\hline \multirow[t]{2}{*}{ MED3 } & \multirow{2}{*}{$\begin{array}{l}\text { Peugeot } \\
406 \text { Break }\end{array}$} & \multirow[t]{2}{*}{1994} & \multirow[t]{2}{*}{1454} & \multirow{2}{*}{$\begin{array}{c}\text { Corner } \\
\text { Calorimeter }\end{array}$} & \multirow[t]{2}{*}{ N/A } & \multirow{2}{*}{$\begin{array}{c}1.5 \mathrm{~L} \\
\text { gasoline } \\
\text { in open } \\
\text { tray }\end{array}$} & \multirow{2}{*}{$\begin{array}{c}\text { Under } \\
\text { gear box }\end{array}$} & \multirow[t]{2}{*}{ Included } & \multirow[t]{2}{*}{ Y } & \multirow[t]{2}{*}{$N$} & \multirow{2}{*}{$\begin{array}{l}\text { Oxygen } \\
\text { depletion }\end{array}$} & $\mathrm{N}$ & (Joyeux et al. 2002) \\
\hline & & & & & & & & & & & & & ED 1995 \\
\hline \multirow[t]{2}{*}{ MED4 } & Unknown & Undetermined, & 1470 & Open & Driver and & Cloth & Driver's & Included & $N$ & $N$ & Oxygen & $\mathrm{N}$ & (Shintani et al. 2004) \\
\hline & & $\begin{array}{l}\text { available from } \\
1970 \text { - late } \\
1990 \text { s }\end{array}$ & & Calorimeter & $\begin{array}{l}\text { passenger } \\
\text { windows rolled } \\
\text { down } 10 \mathrm{~cm}\end{array}$ & $\begin{array}{l}\text { soaked } \\
\text { with } \\
\text { methanol }\end{array}$ & seat & & & & depletion & & DP March 2004 \\
\hline MED5 & Renault & Undetermined, & $1380-$ & $\mathrm{N} / \mathrm{A}$ & 60 I of fuel was & N/A & $\mathrm{N} / \mathrm{A}$ & Unclear & $\mathrm{N}$ & $\mathrm{N}$ & Not & $N$ & (Marlair et al. 2008) \\
\hline & Laguna & $\begin{array}{c}\text { available from } \\
1993 \text { - } 1999\end{array}$ & 1550 & & in the fuel tank & & & & & & mentıoned & & ED June 1999 \\
\hline
\end{tabular}


Table 8 Passenger car : heavy

\begin{tabular}{|c|c|c|c|c|c|c|c|c|c|c|c|c|c|}
\hline ID & $\begin{array}{c}\text { Vehicle } \\
\text { make } \\
\text { and model }\end{array}$ & Vehicle year & $\begin{array}{c}\text { Curb } \\
\text { weight } \\
\text { (kg) }\end{array}$ & $\begin{array}{l}\text { Facility } \\
\text { type }\end{array}$ & Condition & $\begin{array}{l}\text { Ignition } \\
\text { source }\end{array}$ & $\begin{array}{l}\text { Ignition } \\
\text { location }\end{array}$ & $\begin{array}{l}\text { Incipient } \\
\text { stage }\end{array}$ & $\begin{array}{l}\text { Mass } \\
\text { loss } \\
\text { rate }\end{array}$ & $\begin{array}{c}\text { Carbon emission/ } \\
\text { Product } \\
\text { consumption }\end{array}$ & $\begin{array}{l}\text { Heat release } \\
\text { rate evaluation } \\
\text { method }\end{array}$ & $\begin{array}{c}\text { Smoke } \\
\text { production }\end{array}$ & $\begin{array}{c}\text { Reference and } \\
\text { experiment date (ED)/ } \\
\text { report submitted (RS)/ } \\
\text { date published (DP) }\end{array}$ \\
\hline \multirow[t]{2}{*}{$\mathrm{H} 1$} & \multirow{2}{*}{$\begin{array}{l}\text { Honda } \\
\text { Accord }\end{array}$} & \multirow[t]{2}{*}{1998} & \multirow[t]{2}{*}{1649} & \multirow{2}{*}{$\begin{array}{c}\text { Open } \\
\text { Calorimeter }\end{array}$} & \multirow{2}{*}{$\begin{array}{l}\text { All doors } \\
\text { closed and front } \\
\text { door windows } \\
\text { raised, left and } \\
\text { right rear door } \\
\text { glass broken }\end{array}$} & \multirow{2}{*}{$\begin{array}{c}\text { Pool from } \\
400 \mathrm{~mL} / \mathrm{min} \\
\text { fuel tank leak } \\
\text { ignited at } 35 \mathrm{~s}\end{array}$} & \multirow{2}{*}{$\begin{array}{l}\text { Under } \\
\text { vehicle }\end{array}$} & \multirow[t]{2}{*}{ Included } & \multirow[t]{2}{*}{ Y } & \multirow{2}{*}{$\begin{array}{l}\mathrm{Y}\left(\mathrm{CO} \& \mathrm{CO}_{2}\right. \\
\text { production) }\end{array}$} & \multirow{2}{*}{$\begin{array}{l}\text { Species-based } \\
\text { calorimetry \& } \\
\text { other methods }\end{array}$} & \multirow[t]{2}{*}{ Y } & (Santrock 2003a) \\
\hline & & & & & & & & & & & & & ED 25 Feb 1999 \\
\hline \multirow[t]{2}{*}{$\mathrm{H} 2$} & \multirow{2}{*}{$\begin{array}{l}\text { Honda } \\
\text { Accord }\end{array}$} & \multirow[t]{2}{*}{1998} & \multirow[t]{2}{*}{1738} & \multirow{2}{*}{$\begin{array}{c}\text { Open } \\
\text { Calorimeter }\end{array}$} & \multirow{2}{*}{$\begin{array}{l}\text { Windshield and } \\
\text { right front door } \\
\text { glass broken }\end{array}$} & \multirow[t]{2}{*}{ Methanol vapour } & \multirow{2}{*}{$\begin{array}{l}\text { Windshield } \\
\text { washer fluid } \\
\text { reservoir }\end{array}$} & \multirow[t]{2}{*}{ Included } & \multirow[t]{2}{*}{ Y } & \multirow{2}{*}{$\begin{array}{l}\mathrm{Y}\left(\mathrm{CO} \& \mathrm{CO}_{2}\right. \\
\text { production) }\end{array}$} & \multirow{2}{*}{$\begin{array}{l}\text { Species-based } \\
\text { calorimetry \& } \\
\text { other methods }\end{array}$} & \multirow[t]{2}{*}{ Y } & (Santrock 2003b) \\
\hline & & & & & & & & & & & & & ED 23 Feb 1999 \\
\hline \multirow[t]{2}{*}{ H3 } & \multirow{2}{*}{$\begin{array}{l}\text { Chevrolet } \\
\text { Camaro }\end{array}$} & \multirow[t]{2}{*}{1997} & \multirow[t]{2}{*}{1811} & \multirow{2}{*}{$\begin{array}{c}\text { Open } \\
\text { Calorimeter }\end{array}$} & \multirow[b]{2}{*}{$\begin{array}{l}\text { Left side } \\
\text { door window and } \\
\text { rear compartment } \\
\text { lift window were } \\
\text { shattered, gap } \\
\text { between the bottom } \\
\text { of the left door } \\
\text { and frame }\end{array}$} & \multirow{2}{*}{$\begin{array}{l}\text { Pool from } \\
515 \mathrm{~mL} / \mathrm{min} \\
\text { fuel tank leak } \\
\text { ignited at } 30 \mathrm{~s}\end{array}$} & \multirow{2}{*}{$\begin{array}{l}\text { Under } \\
\text { vehicle }\end{array}$} & \multirow[t]{2}{*}{ Included } & Y & $Y\left(\mathrm{CO} \& \mathrm{CO}_{2}\right.$ & Species-based & Y & (Santrock 2002d) \\
\hline & & & & & & & & & & production) & $\begin{array}{l}\text { calorimetry \& } \\
\text { other methods }\end{array}$ & & ED 30 Sep 1997 \\
\hline $\mathrm{H} 4$ & Chevrolet & 1999 & 1848 & Open & Doors closed with & Nichrome wires & In air cleaner & Included & Y & $\mathrm{Y}\left(\mathrm{CO} \& \mathrm{CO}_{2}\right.$ & Species-based & Y & (Santrock 2002a) \\
\hline & (Modified) & & & & $\begin{array}{l}\text { window glass } \\
\text { (passenger door) of } \\
\text { the vehicle broken }\end{array}$ & PP sheet (1.2 kW) & $\begin{array}{l}\text { nousing in } \\
\text { engine } \\
\text { compartment }\end{array}$ & & & & other methods & & ED 21 Feb 2000 \\
\hline H5 & Chevrolet & 1999 & 1848 & Open & Doors closed with & Nichrome wires & In air cleaner & Included & Y & $\mathrm{Y}\left(\mathrm{CO} \& \mathrm{CO}_{2}\right.$ & Species-based & Y & (Santrock 2002a) \\
\hline & & & & Calorimeter & $\begin{array}{l}\text { windows raised to full } \\
\text { closed position, right } \\
\text { window glass } \\
\text { (passenger door) of } \\
\text { the vehicle broken }\end{array}$ & $\begin{array}{l}\text { Wrapped around } \\
\text { PP sheet (1.2 kW) }\end{array}$ & $\begin{array}{l}\text { housing in } \\
\text { engine } \\
\text { compartment }\end{array}$ & & & production) & $\begin{array}{l}\text { calorimetry \& } \\
\text { other methods }\end{array}$ & & ED 21 Feb 2000 \\
\hline H6 & Chevrolet & 1997 & 1849 & Open & Windshield and right & Propane torch & Engine & Included & Y & $\mathrm{Y}\left(\mathrm{CO} \& \mathrm{CO}_{2}\right.$ & Species-based & Y & (Santrock 2002e) \\
\hline & & & & & $\begin{array}{l}\text { door window were } \\
\text { broken and a section } \\
\text { of the weld seam } \\
\text { between the floor pan } \\
\text { and inner rocker panel } \\
\text { was separated }\end{array}$ & $\begin{array}{l}\text { flame impinging } \\
\text { on HVAC } \\
\text { module }\end{array}$ & compartment & & & & $\begin{array}{l}\text { calorimetry \& } \\
\text { other methods }\end{array}$ & & ED 1 Oct 1997 \\
\hline $\mathrm{H7}$ & Unknown & Undetermined, & 1920 & Open & Driver and passenger & Cloth soaked & Driver's seat & Included & N & $N$ & Oxygen & $N$ & (Shintani et al. 2004) \\
\hline & & $\begin{array}{c}\text { avallable from } \\
1970-\text { late } \\
\text { 1990s }\end{array}$ & & & $\begin{array}{l}\text { Windows rolled down } \\
\qquad 10 \mathrm{~cm}\end{array}$ & & & & & & & & DP March 2004 \\
\hline
\end{tabular}


Table 9 Sport-utility vehicle (SUV)

\begin{tabular}{|c|c|c|c|c|c|c|c|c|c|c|c|c|c|}
\hline ID & $\begin{array}{l}\text { Vehicle } \\
\text { make and } \\
\text { model }\end{array}$ & $\begin{array}{l}\text { Vehicle } \\
\text { year }\end{array}$ & $\begin{array}{l}\text { Curb } \\
\text { weight } \\
\text { (kg) }\end{array}$ & $\begin{array}{l}\text { Facility } \\
\text { type }\end{array}$ & Condition & $\begin{array}{l}\text { Ignition } \\
\text { source }\end{array}$ & $\begin{array}{l}\text { Ignition } \\
\text { location }\end{array}$ & $\begin{array}{l}\text { Incipient } \\
\text { stage }\end{array}$ & $\begin{array}{l}\text { Mass } \\
\text { loss } \\
\text { rate }\end{array}$ & $\begin{array}{l}\text { Carbon emission/ } \\
\text { Product consumption }\end{array}$ & $\begin{array}{l}\text { Heat release } \\
\text { rate evalution } \\
\text { method }\end{array}$ & $\begin{array}{c}\text { Smoke } \\
\text { production }\end{array}$ & $\begin{array}{c}\text { Reference and } \\
\text { experiment date (ED)/ } \\
\text { report submitted (RS)/ } \\
\text { date published (DP) }\end{array}$ \\
\hline \multirow[t]{2}{*}{ SUV1 } & \multirow{2}{*}{$\begin{array}{l}\text { Ford } \\
\text { Explorer }\end{array}$} & \multirow[t]{2}{*}{1998} & \multirow[t]{2}{*}{2232} & \multirow{2}{*}{$\begin{array}{c}\text { Open } \\
\text { Calorimeter }\end{array}$} & \multirow{2}{*}{$\begin{array}{l}\text { Pass through } \\
\text { openings under left front } \\
\text { seat; shift lever; drain } \\
\text { holes, left door and } \\
\text { door sills }\end{array}$} & \multirow{2}{*}{$\begin{array}{l}\text { Pool from } \\
350 \mathrm{~mL} / \mathrm{min} \\
\text { fuel tank leak } \\
\text { ignited at } 30 \mathrm{~s}\end{array}$} & \multirow{2}{*}{$\begin{array}{l}\text { Under vehicle } \\
\text { (mid-body) }\end{array}$} & \multirow{2}{*}{ Included } & \multirow[t]{2}{*}{ Y } & \multirow{2}{*}{$\begin{array}{l}\mathrm{Y}\left(\mathrm{CO} \& \mathrm{CO}_{2}\right. \\
\text { production) }\end{array}$} & \multirow{2}{*}{$\begin{array}{l}\text { Species-based } \\
\text { calorimetry \& } \\
\text { other methods }\end{array}$} & \multirow[t]{2}{*}{ Y } & (Santrock 2002b) \\
\hline & & & & & & & & & & & & & ED 11 Jun 1998 \\
\hline \multirow[t]{2}{*}{ SUV2 } & \multirow{2}{*}{$\begin{array}{l}\text { Ford } \\
\text { Explorer }\end{array}$} & \multirow[t]{2}{*}{1998} & \multirow[t]{2}{*}{2249} & \multirow{2}{*}{$\begin{array}{c}\text { Open } \\
\text { Calorimeter }\end{array}$} & \multirow{2}{*}{$\begin{array}{l}\text { Window openings on } \\
\text { the left and right quarter } \\
\text { panels; additional opening on } \\
\text { the rear lift gate, left rear door, } \\
\text { door frames and seams } \\
\text { along the rear } \\
\text { compartment floor panels }\end{array}$} & \multirow{2}{*}{$\begin{array}{l}\text { Pool from } \\
750 \mathrm{~mL} / \mathrm{min} \\
\text { fuel tank leak } \\
\text { ignited at } 30 \mathrm{~s}\end{array}$} & \multirow{2}{*}{ Under vehicle } & \multirow[t]{2}{*}{ Included } & \multirow[t]{2}{*}{ Y } & \multirow{2}{*}{$\begin{array}{l}\mathrm{Y}\left(\mathrm{CO} \& \mathrm{CO}_{2}\right. \\
\text { production) }\end{array}$} & \multirow{2}{*}{$\begin{array}{l}\text { Species-based } \\
\text { calorimetry \& } \\
\text { other methods }\end{array}$} & \multirow[t]{2}{*}{ Y } & (Santrock 2002f) \\
\hline & & & & & & & & & & & & & ED 9 Jun 1998 \\
\hline
\end{tabular}


Table 10 Multi-purpose vehicle (MPV)

\begin{tabular}{|c|c|c|c|c|c|c|c|c|c|c|c|c|c|}
\hline ID & $\begin{array}{l}\text { Vehicle make } \\
\text { and model }\end{array}$ & Vehicle year & $\begin{array}{c}\text { Curb } \\
\text { weight }(\mathrm{kg})\end{array}$ & $\begin{array}{l}\text { Facility } \\
\text { type }\end{array}$ & Condition & $\begin{array}{l}\text { Ignition } \\
\text { source }\end{array}$ & $\begin{array}{l}\text { Ignition } \\
\text { location }\end{array}$ & $\begin{array}{l}\text { Incipient } \\
\text { stage }\end{array}$ & $\begin{array}{c}\text { Mass } \\
\text { loss rate }\end{array}$ & $\begin{array}{c}\text { Carbon } \\
\text { emission/Product } \\
\text { consumption }\end{array}$ & $\begin{array}{l}\text { Heat release } \\
\text { rate evaluation } \\
\text { method }\end{array}$ & $\begin{array}{c}\text { Smoke } \\
\text { production }\end{array}$ & $\begin{array}{c}\text { Reference and } \\
\text { experiment date (ED)/ } \\
\text { report submitted (RS)/ } \\
\text { date published (DP) }\end{array}$ \\
\hline \multirow[t]{2}{*}{ MPV1 } & \multirow{2}{*}{$\begin{array}{l}\text { Plymouth } \\
\text { Voyager }\end{array}$} & \multirow[t]{2}{*}{1996} & \multirow[t]{2}{*}{1946} & \multirow{2}{*}{$\begin{array}{c}\text { Open } \\
\text { Calorimeter }\end{array}$} & \multirow[b]{2}{*}{$\begin{array}{l}\text { Rear hatch } \\
\text { window } \\
\text { broken, left } \\
\text { rear vent } \\
\text { window } \\
\text { open, left } \\
\text { rear quarter } \\
\text { panel } \\
\text { cracked } \\
\text { from crash }\end{array}$} & \multirow[b]{2}{*}{$\begin{array}{c}\text { Pool } \\
\text { from } 243 \\
\mathrm{ML} / \mathrm{min} \\
\text { fuel tank } \\
\text { leak } \\
\text { ignited } \\
\text { at } 30 \mathrm{~s}\end{array}$} & \multirow{2}{*}{$\begin{array}{l}\text { Under } \\
\text { vehicle }\end{array}$} & \multirow[t]{2}{*}{ Included } & \multirow[t]{2}{*}{ Y } & \multirow{2}{*}{$\begin{array}{l}\mathrm{Y}\left(\mathrm{CO} \& \mathrm{CO}_{2}\right. \\
\text { production) }\end{array}$} & \multirow{2}{*}{$\begin{array}{l}\text { Species-based } \\
\text { calorimetry \& } \\
\text { other methods }\end{array}$} & \multirow[t]{2}{*}{ Y } & (Santrock 2002c) \\
\hline & & & & & & & & & & & & & ED 15 Nov 1996 \\
\hline \multirow[t]{2}{*}{ MPV2 } & \multirow{2}{*}{$\begin{array}{l}\text { Dodge } \\
\text { Caravan Sport }\end{array}$} & \multirow[t]{2}{*}{1996} & \multirow[t]{2}{*}{1981} & \multirow{2}{*}{$\begin{array}{l}\text { Open } \\
\text { Calorimeter }\end{array}$} & \multirow{2}{*}{$\begin{array}{l}\text { Driver and } \\
\text { passenger } \\
\text { window } \\
\text { slightly } \\
\text { open }\end{array}$} & \multirow{2}{*}{$\begin{array}{l}\text { Electrical } \\
\text { wire } \\
\text { igniter }\end{array}$} & \multirow{2}{*}{$\begin{array}{l}\text { Around } \\
\text { battery and } \\
\text { power } \\
\text { distributor } \\
\text { housing }\end{array}$} & \multirow[t]{2}{*}{ Included } & \multirow[t]{2}{*}{ Y } & \multirow{2}{*}{$\begin{array}{l}\mathrm{Y}\left(\mathrm{CO} \& \mathrm{CO}_{2}\right. \\
\text { production) }\end{array}$} & \multirow{2}{*}{$\begin{array}{l}\text { Species-based } \\
\text { calorimetry \& } \\
\text { other methods }\end{array}$} & \multirow[t]{2}{*}{ Y } & (Santrock 2001) \\
\hline & & & & & & & & & & & & & ED 13 Nov 1996 \\
\hline \multirow[t]{2}{*}{ MPV3 } & \multirow{2}{*}{$\begin{array}{l}\text { Unknown } \\
\text { (Minivan) }\end{array}$} & \multirow[t]{2}{*}{1995} & \multirow[t]{2}{*}{ N/A } & \multirow{2}{*}{$\begin{array}{c}\text { Open } \\
\text { Calorimeter }\end{array}$} & \multirow{2}{*}{$\begin{array}{l}\text { Driver and } \\
\text { passenger } \\
\text { window } \\
\text { open }\end{array}$} & \multirow{2}{*}{$\begin{array}{l}2 \mathrm{~L} \text { of } \\
\text { gasoline }\end{array}$} & \multirow{2}{*}{$\begin{array}{l}\text { Poured on } \\
\text { driver's seat }\end{array}$} & \multirow{2}{*}{$\begin{array}{l}\text { Included } \\
\text { (prior } \\
\text { experiment } \\
\text { not } \\
\text { included) }\end{array}$} & \multirow[t]{2}{*}{$\mathrm{N}$} & $\mathrm{Y}\left(\mathrm{O}_{2}\right.$ & Temperature & $\mathrm{N}$ & (Stroup et al. 2001) \\
\hline & & & & & & & & & & $\begin{array}{l}\text { consumption, } \mathrm{CO} \\
\& \mathrm{CO}_{2} \text { production) }\end{array}$ & difference & & ED 7 Dec 1999 \\
\hline MPV4 & Renault & Undetermined, & $1170-1780$ & Parking & N/A & N/A & N/A & Unclear & Y & $\mathrm{N}$ & Mass loss & N & (Van Oerle et al. 1999) \\
\hline & Espace & $\begin{array}{c}\text { available from } \\
\text { 1984 - Late } \\
\text { 1990s }\end{array}$ & & Garage & & & & & & & & & DP 5 Nov 1999 \\
\hline MPV5 & Renault & 2001 & $1170-1780$ & Room & & IMS & Engine & Included & $\mathrm{N}$ & $\mathrm{N}$ & Species-based & N & (CLG 2010) \\
\hline & & & & & $\begin{array}{c}\text { passenger } \\
\text { windows } \\
\text { closed, } \\
\text { bonnet } \\
\text { closed after } \\
\text { fire has } \\
\text { established. }\end{array}$ & $\begin{array}{l}\text { Soaked } \\
\text { fibre- } \\
\text { board }\end{array}$ & compartment & & & & & & ED 1 Sep 2008 \\
\hline MPV6 & Renault & Undetermined, & $1170-1780$ & Tunnel & N/A & N/A & N/A & Unclear & $\mathrm{N}$ & N & Species-based & N & (Steinert 1994) \\
\hline & & $\begin{array}{l}\text { avallable from } \\
1984 \text { - } 1994\end{array}$ & & & & & & & & & & & DP 1994 \\
\hline
\end{tabular}


Table 11 Unclassfied vehicles

\begin{tabular}{|c|c|c|c|c|c|c|c|c|c|c|c|c|c|}
\hline ID & $\begin{array}{l}\text { Vehicle make } \\
\text { and model }\end{array}$ & $\begin{array}{l}\text { Vehicle } \\
\text { year }\end{array}$ & $\begin{array}{c}\text { Curb } \\
\text { weight (kg) }\end{array}$ & $\begin{array}{l}\text { Facility } \\
\text { type }\end{array}$ & Condition & $\begin{array}{l}\text { Ignition } \\
\text { source }\end{array}$ & $\begin{array}{l}\text { Ignition } \\
\text { location }\end{array}$ & $\begin{array}{l}\text { Incipient } \\
\text { stage }\end{array}$ & $\begin{array}{c}\text { Mass } \\
\text { loss rate }\end{array}$ & $\begin{array}{l}\text { Carbon emission/ } \\
\text { Product } \\
\text { consumption }\end{array}$ & $\begin{array}{l}\text { Heat release } \\
\text { rate evaluation } \\
\text { method }\end{array}$ & $\begin{array}{c}\text { Smoke } \\
\text { production }\end{array}$ & $\begin{array}{c}\text { Reference and } \\
\text { experiment date (ED)/ } \\
\text { report submitted (RS)/ } \\
\text { date published (DP) }\end{array}$ \\
\hline \multirow[t]{2}{*}{ U1 } & \multirow[t]{2}{*}{ Unknown } & \multirow[t]{2}{*}{1998} & \multirow[t]{2}{*}{ N/A } & \multirow[t]{2}{*}{$\begin{array}{c}\text { Open } \\
\text { Calorimeter }\end{array}$} & \multirow{2}{*}{$\begin{array}{c}\text { Battery removed, } \\
\text { petrol tank } \\
\text { emptied, air bags, } \\
\text { belt stretchers, } \\
\text { side impact } \\
\text { protection inactivated. }\end{array}$} & \multirow{2}{*}{$\begin{array}{l}0.21 \mathrm{~L} \\
\text { of mineral } \\
\text { spirits }\end{array}$} & \multirow{2}{*}{$\begin{array}{l}\text { On driver's } \\
\text { seat and } \\
\text { right rear } \\
\text { passenger seat }\end{array}$} & \multirow[t]{2}{*}{ Included } & \multirow[t]{2}{*}{$N$} & \multirow[t]{2}{*}{$\begin{array}{l}\mathrm{Y}\left(\mathrm{CO}, \mathrm{HCN}, \mathrm{HCl} \& \mathrm{SO}_{2}\right. \\
\text { production) }\end{array}$} & \multirow[t]{2}{*}{$\begin{array}{l}\text { Oxygen } \\
\text { depletion }\end{array}$} & \multirow[t]{2}{*}{$N$} & $\begin{array}{l}\text { (Lönnermark and } \\
\text { Blomqvist 2006) }\end{array}$ \\
\hline & & & & & & & & & & & & & RS 21 Dec 2004 \\
\hline
\end{tabular}




\section{Vehicle fires}

In order for fire to occur in a passenger vehicle there are three main elements that must be present. First are the combustible materials which include fluids such as engine fuels and oils, transmission oils, power steering fluids, brake fluids and lubricants; upholsteries; tyres; plastic materials such as in dashboards and bumpers; possibly the body work of the vehicle itself; and finally, any contents being carried in the vehicle. Second is the availability of oxygen depends on whether vehicle doors and windows are open (and/or break during the fire), the ease that air can reach other internal parts of the car plus the external ventilation if the vehicle is in an enclosure. Some of the experiments collated in this review include the difference in vehicle burning characteristics as a result of the degree of opening of the vehicle windows. The third element is the source of ignition. Common sources of ignition for vehicles are electrical faults, hot surfaces, mechanical failure and deliberate actions (Cheong et al. 2008).

The heat release rate curves for single passenger vehicles are obtained from several publications of large-scale calorimeter fire experiments dating back from 1980s to the late 2000s. The review is limited to single vehicles since multiple vehicle experimental data is sparse. From the heat release rate curves the focus is on three characteristics which can be directly obtained from the heat release rate curves; the peak heat release rate, the time to reach peak heat release rate and the total energy released. There is inevitably an overlap with previous work, in particular the study of design fires for vehicles in tunnels by (Ingason 2006) and the database of vehicle fires available from (Janssens 2008). However, these studies did not consider any form of vehicle classification and corresponding statistical analysis of severity a) Experiment M1

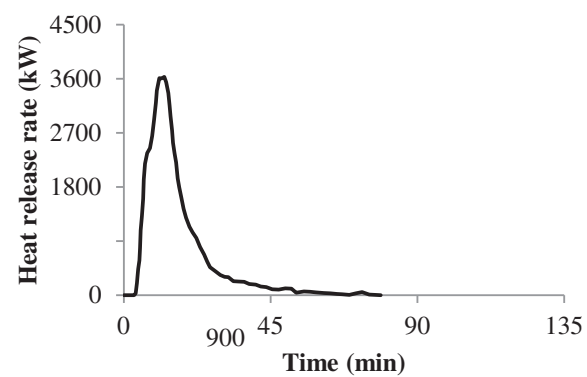

b) Experiment M2

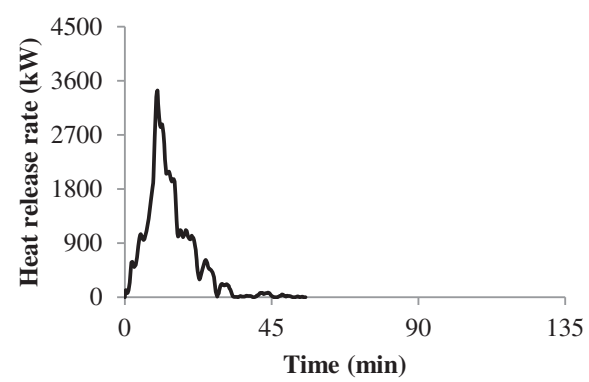

c) Experiment M3

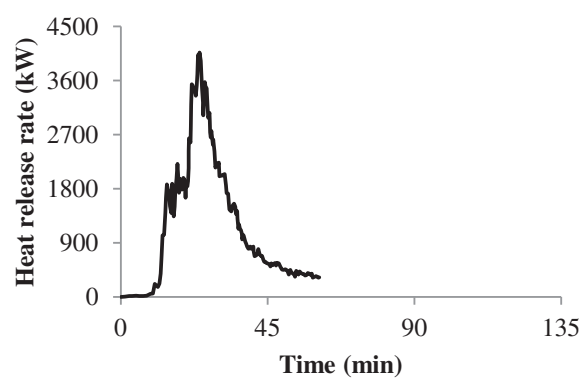

d) Experiment M4

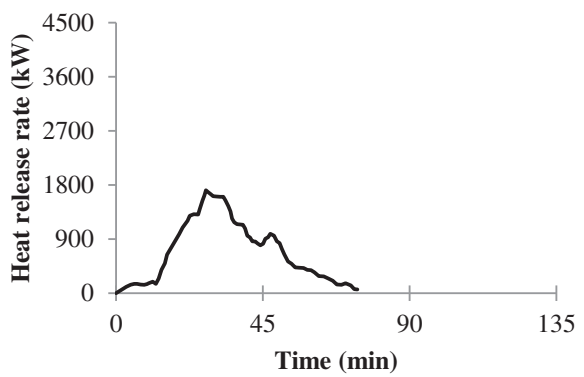

e) Experiment M5

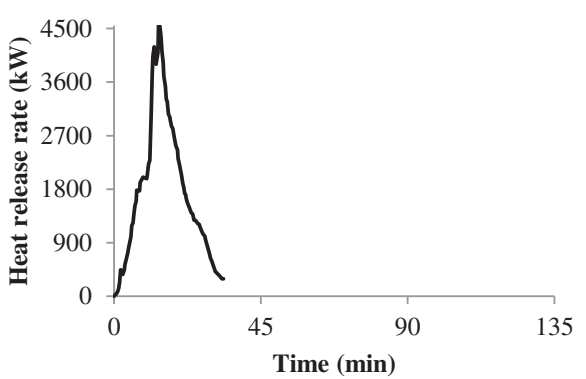

f) Experiment M6

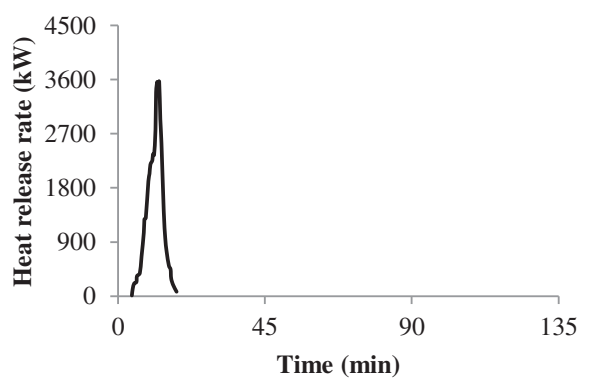

Figure 1 List of experiments; (a) Experiment M1 (b) Experiment M2 (c) Experiment M3 (d) Experiment M4 (e) Experiment M5 (f) Experiment M6. 
characteristics and there are experiments that are included in this study that post-date the work by (Ingason 2006) and (Janssens 2008).

To achieve the objectives of the review, two components are presented. First is a collation and summary of the passenger vehicle fire experiments including an associated reproduction of the rate of heat release curve. Second is the distribution analysis which gathers the experiments into the specified weight-related classifications and suggests a distribution shape for each burning severity characteristic of interest. For this purpose the BestFit capability of the @RISK software (Palisade Corporation 2010) is used to process the data sets.

\section{Collation of experiments}

Summary descriptions

A total of 41 single passenger vehicle fire experiments are collated where details are obtained from the corresponding

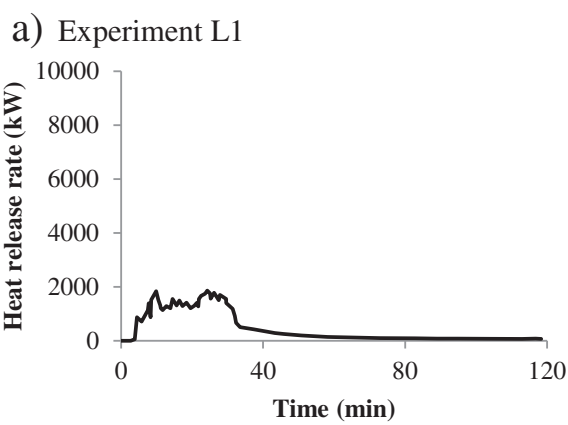

b) Experiment L2

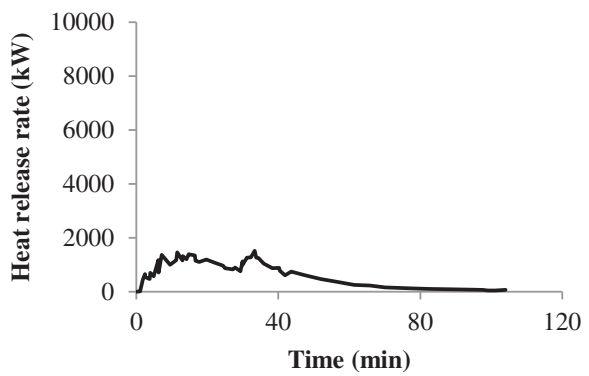

c) Experiment L3

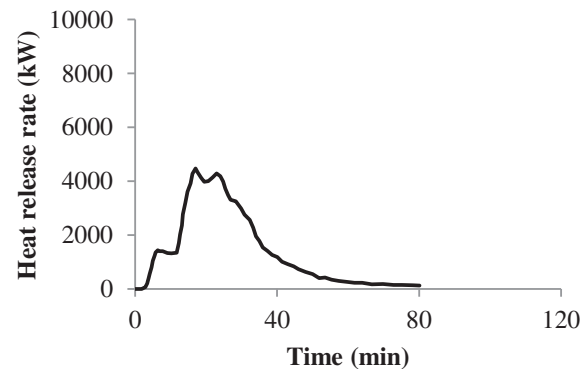

d) Experiment L4

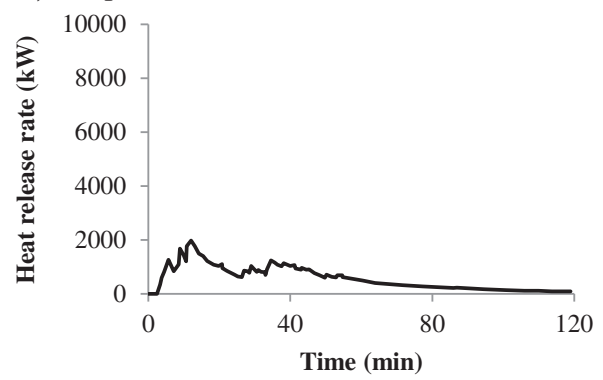

e) Experiment L5

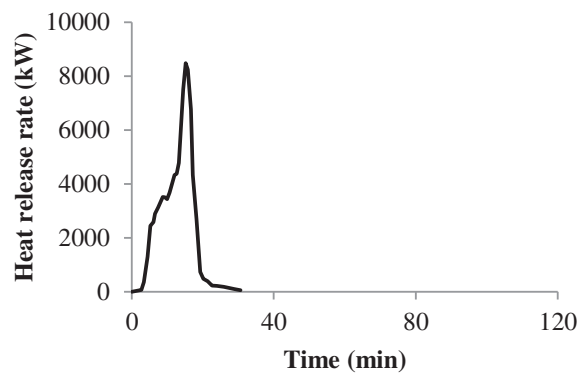

f) Experiment L6

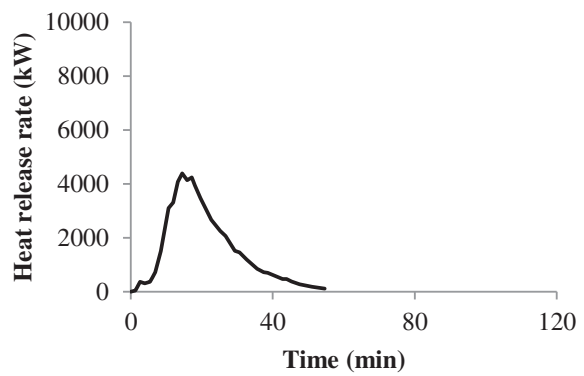

g) Experiment L7

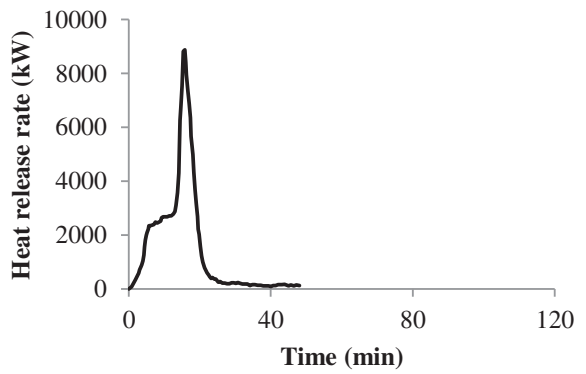

(f) Experiment L6 (g) Experiment L7. 


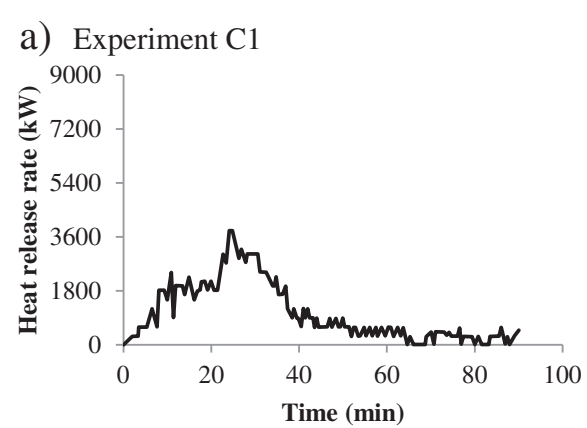

b) Experiment $\mathrm{C} 2$

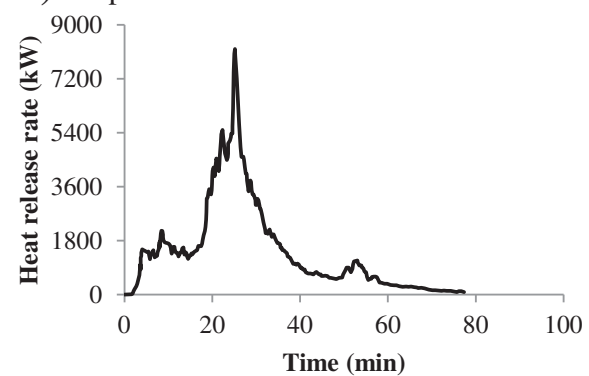

c) Experiment $\mathrm{C} 3$

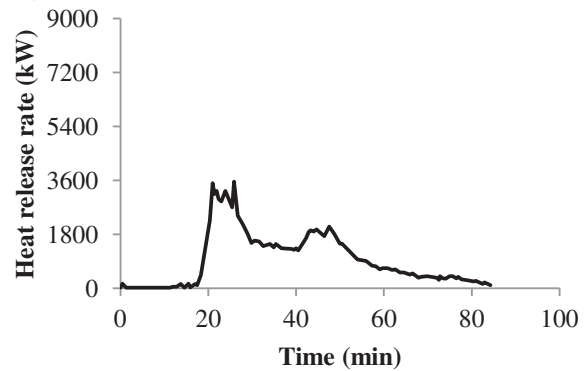

d) Experiment $\mathrm{C} 4$

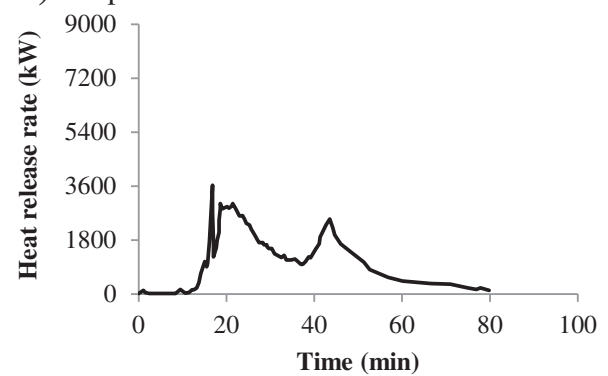

e) Experiment $\mathrm{C5}$

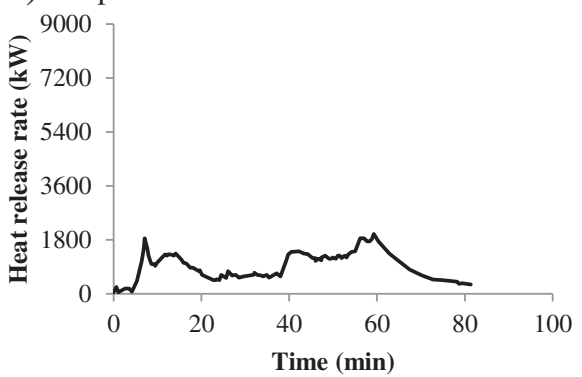

f) Experiment $\mathrm{C6}$

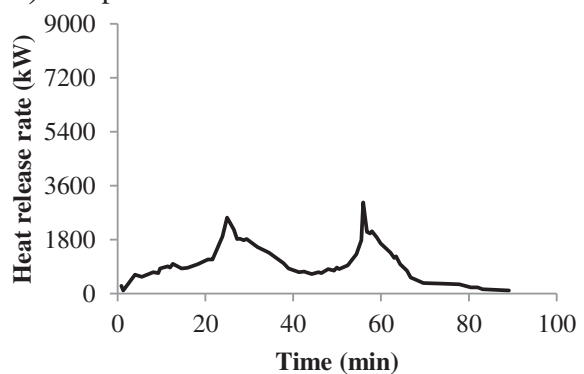

g) Experiment $\mathrm{C} 7$

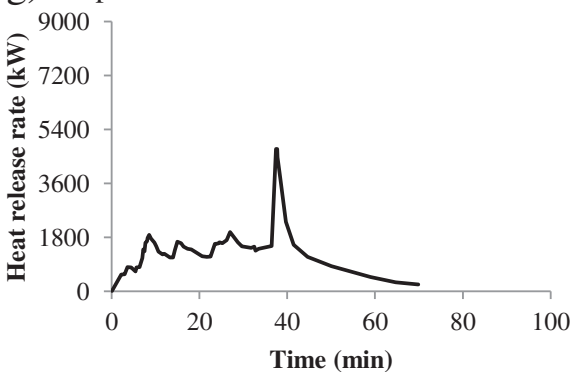

Figure 3 List of experiments; (a) Experiment C1 (b) Experiment C2 (c) Experiment C3 (d) Experiment C4 (e) Experiment C5 (f) Experiment $\mathrm{C} 6$ (g) Experiment $\mathrm{C} 7$.

reference sources. For the ANSI classification, the mass of the vehicle is required but if a source did not explicitly declare the mass then other information is used. For example, where the make, model and the year of manufacture are quoted in the original source, then the mass is obtained from the manufacturer's vehicle specification manual. In some cases the make, model and/or year were not given, thus the mass of the vehicles were obtained using the make and models information from several car specification database websites (Automobile and Car Specifications 2013; Automotive Technical Specifications 2013; Car Technical Specifications 2013). For the references which only specify the general model, a range for the mass of vehicles is collated. In some instances these ranges cross different classification groups and so these experiments are placed into groups which have the majority of the possible mass values for the vehicle model. Table 3 shows the frequency of vehicles 


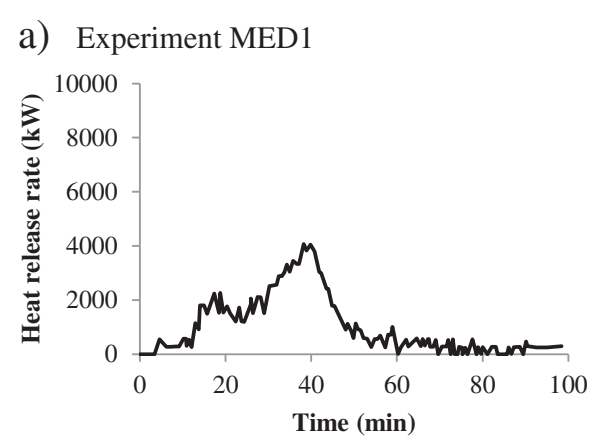

b) Experiment MED2

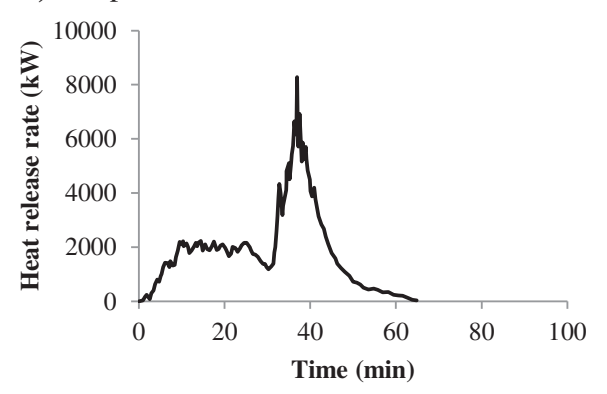

c) Experiment MED3

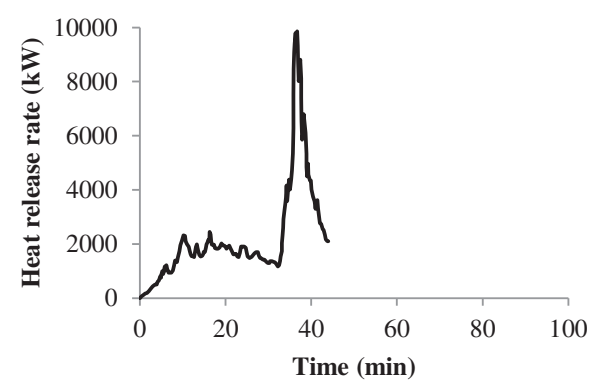

d) Experiment MED4

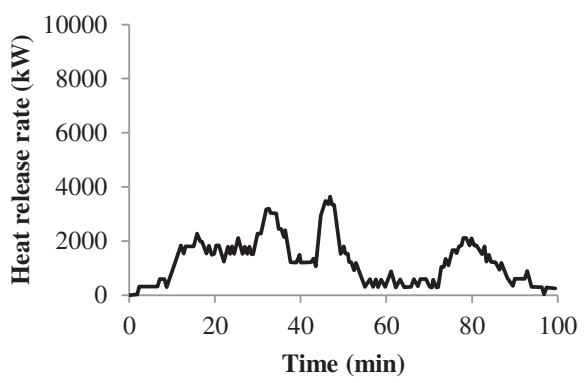

e) Experiment MED5

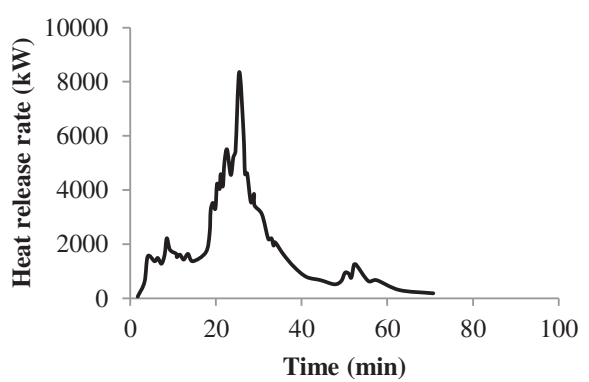

Figure 4 List of experiments; (a) Experiment MED1 (b) Experiment MED2 (c) Experiment MED3 (d) Experiment MED4 (e) Experiment MED5.

when they are grouped using the ANSI classification, however one vehicle is unclassified as there is insufficient detail provided in the original source material. From this point on, the experiments are compiled within its classification for the purpose of comparison and analysis.

Tables 4, 5, 6, 7, 8, 9, 10 and 11 provides a summary of the 41 experiments where each is given a unique identification code related to its ANSI classification which is used throughout this paper rather than the referring to the details of the experiment itself. The exact year of vehicle manufacture not always given in the source reference but typically the decade is identified. In some cases the year of manufacture could only be estimated from the year of the publication of the original source and the years for which the vehicle was available. Values for the mass are that of the vehicle without contents but in some cases the heat release rate data includes the contribution of additional contents included in the experiment. Mass values for experiments M6, M7, L5, L7, MED5, MPV3, MPV4, MPV5 and MPV6 are shown as ranges for reasons explained previously.

The 'Facility type' column is the type of calorimeter used which is most cases was either an open calorimeter that did not restrict air flow to the vehicle or a room calorimeter with limited ventilation paths. The 'Heat release rate evaluation method' column provides information on how the heat release rate curve was obtained from the experiment. However not all references were clear on the exact technique used and so the information provided here is limited by what can be interpreted from the original publication. 'Mass loss' indicates that a mass loss measurement technique was used; 'Convective calorimetry' means the heat release rate was established by using temperature measurements; 'Species-based calorimetry' 


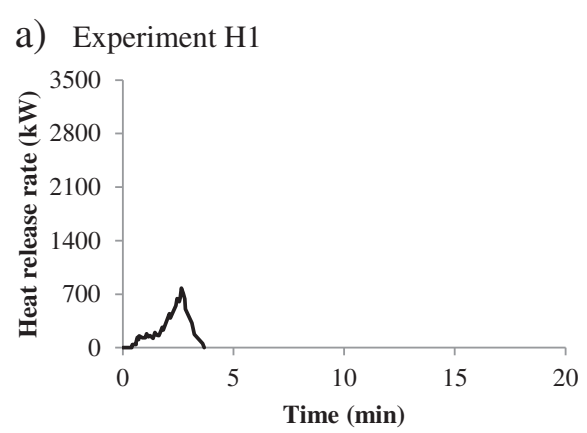

b) Experiment $\mathrm{H} 2$

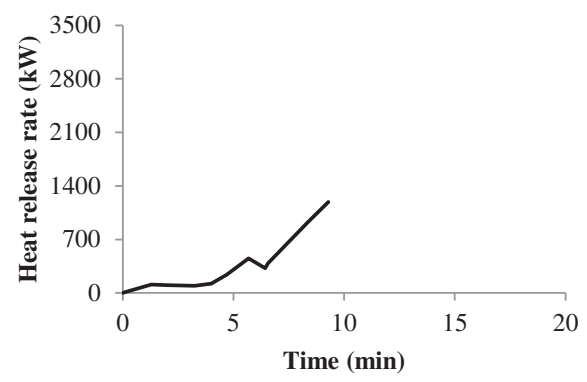

c) Experiment $\mathrm{H} 3$

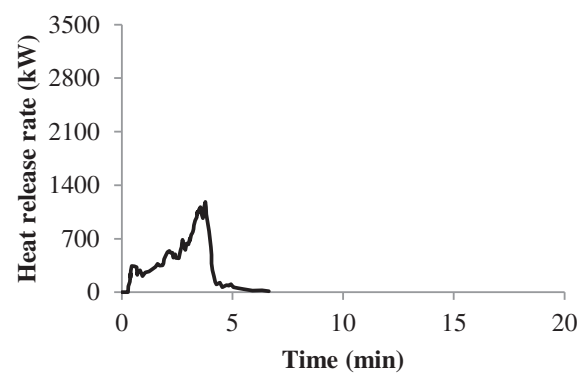

d) Experiment $\mathrm{H} 4$

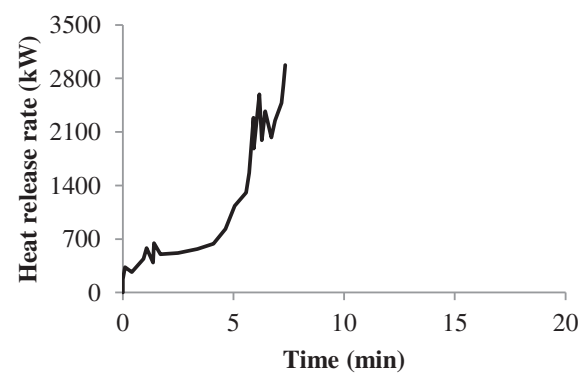

e) Experiment $\mathrm{H} 5$

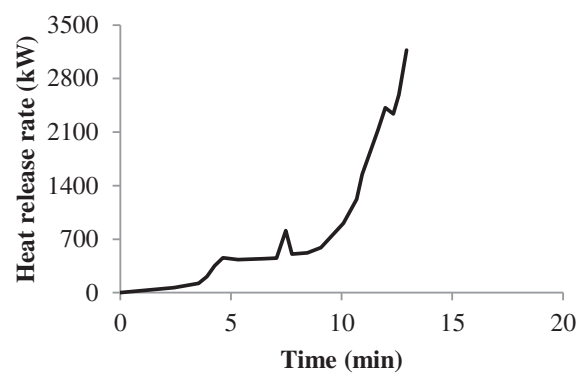

f) Experiment $\mathrm{H} 6$

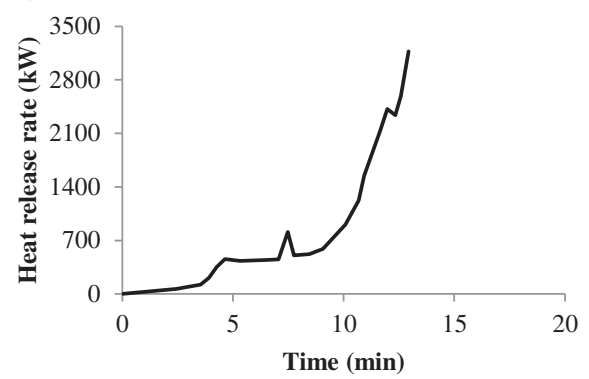

g) Experiment $\mathrm{H} 7$

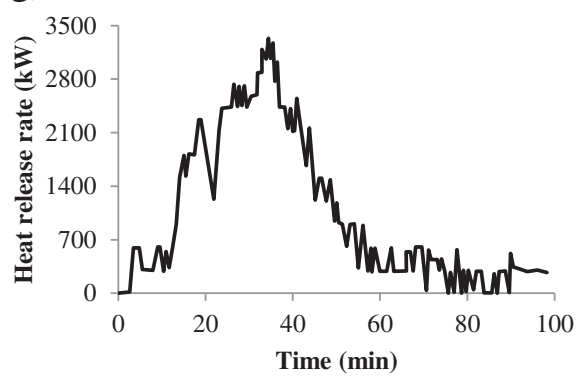

(f) Experiment $\mathrm{H} 6$ (g) Experiment $\mathrm{H} 7$.

means that the heat release rate was obtained using either $\mathrm{O}_{2}$ depletion, $\mathrm{CO}_{2}$ and/or $\mathrm{CO}$ generation while 'Oxygen depletion' means that the use of $\mathrm{O}_{2}$ depletion was clearly stated in the reference. 'Other methods' means that the heat release rate was obtained either using chemical or radiative methods. 'Not mentioned' in the column means that it was unclear what method was used to evaluate the heat release rate curve.

The 'Condition' column provides some detail regarding the vehicle before the fire was started, in particular the degree of openness of any doors and/or windows. The 'Ignition source' column is the additional fuel used to 


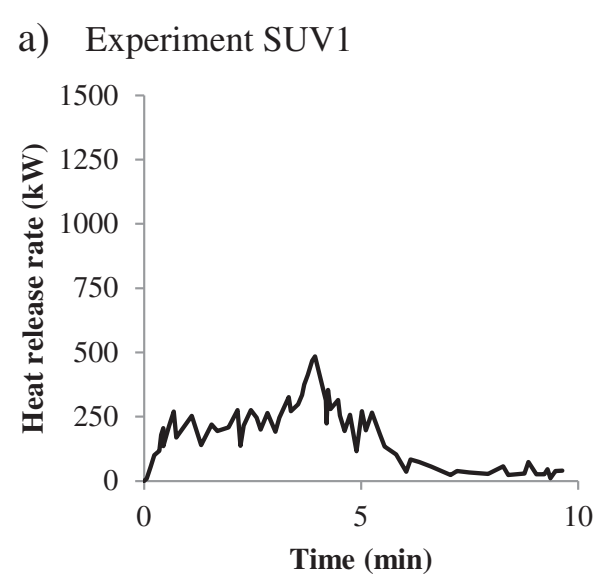

b) Experiment SUV2

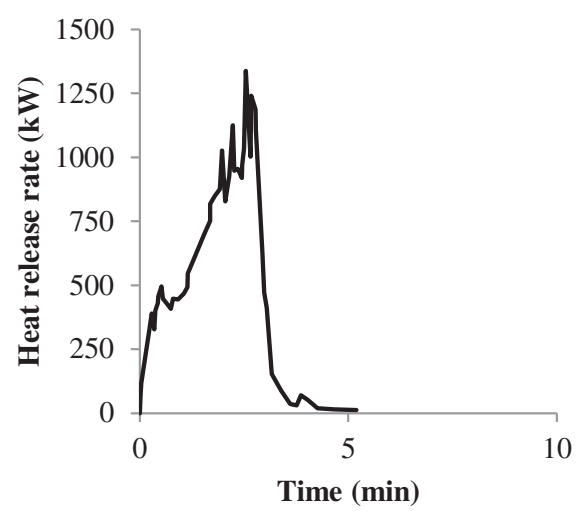

Figure 6 List of experiments; (a) Experiment SUV1 (b) Experiment SUV2.

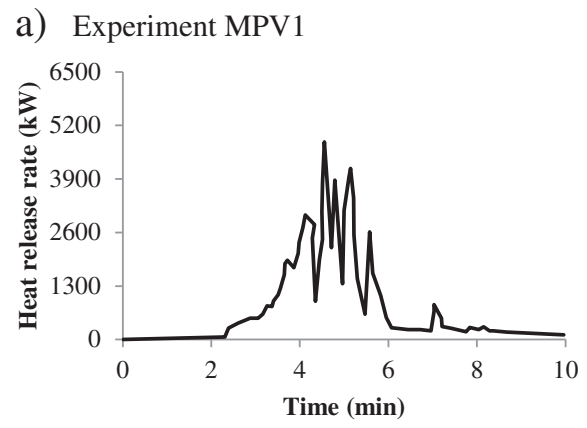

b) Experiment MPV2

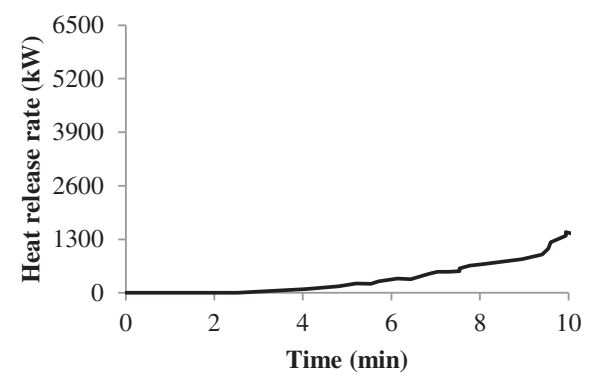

c) Experiment MPV3

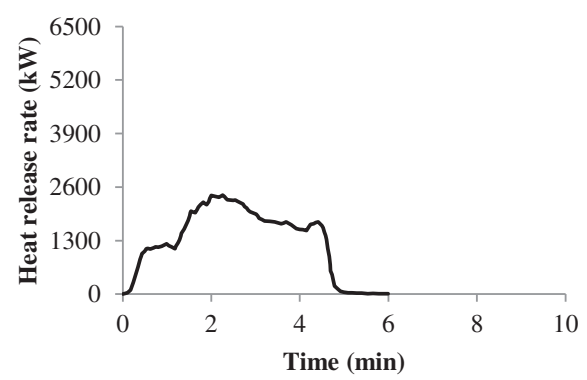

d) Experiment MPV4

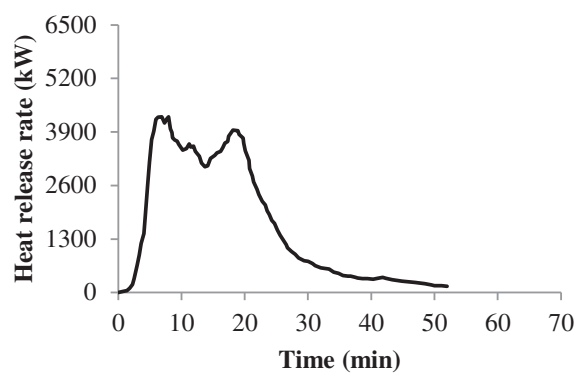

e) Experiment MPV5

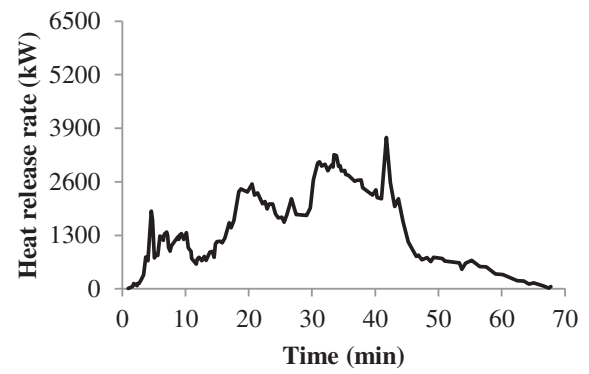

f) Experiment MPV6

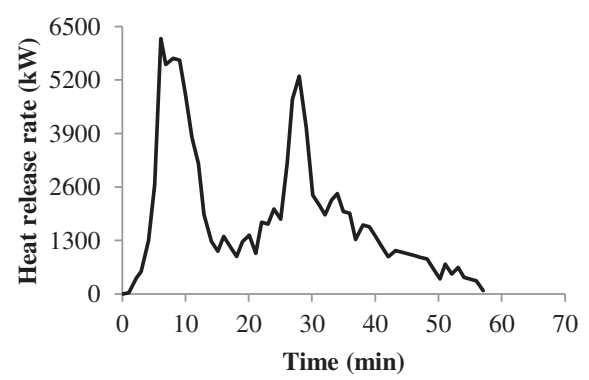

Figure 7 List of experiments; (a) Experiment MPV1 (b) Experiment MPV2 (c) Experiment MPV3 (d) Experiment MPV4 (e) Experiment MPV5 (f) Experiment MPV6. 


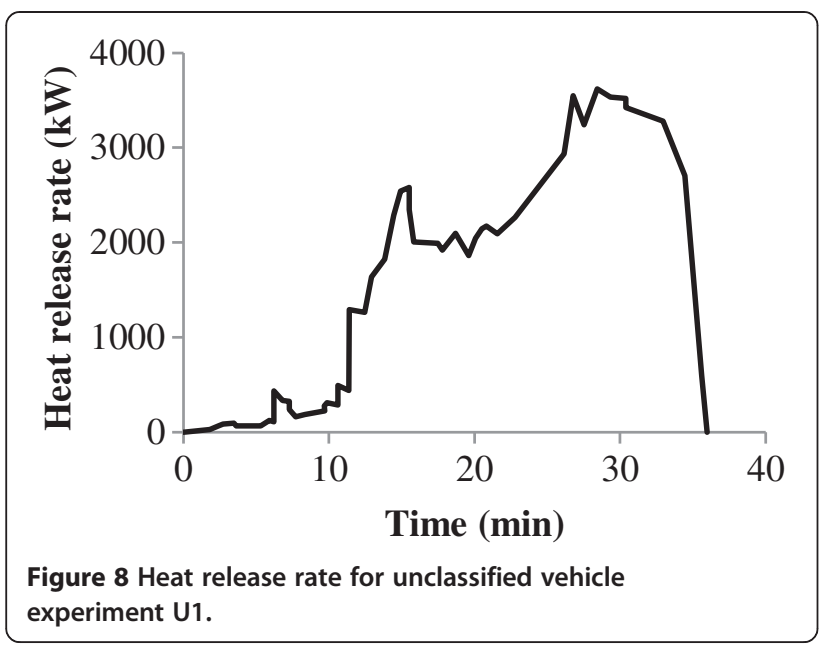

start the fire while the 'Ignition location' column is the point of fire origin. Some of the resources have included the incipient stage in the heat release rate curves and this is clearly identified while for others the inclusion of an incipient stage is not clearly stated or no mention of the incipient stage is made. The status of the incipient stage is indicated in the specified column.

'Mass loss rate' relates to the rate of mass loss during the burning of the vehicle. 'Toxic product emission' relates to information regarding the toxic products produced or emitted during the experiment. The 'smoke production' relates to information regarding the production of smoke from the experiment. For these three items of information ' $\mathrm{Y}$ ' in the column means that information is available in the resource while ' $\mathrm{N}$ ' means that no information is available. The 'Reference and experiment date (ED), report submitted (RS) or date published (DP)' column is the information about the primary reference and about when the experiment was performed, or where no information is given then the date of the report was submitted is listed. If no experiment date and report submission date is available then the published date of the resource is shown.

Table 12 Passenger car : mini

\begin{tabular}{lcccc}
\hline Experiments & $\begin{array}{c}\text { Peak heat } \\
\text { release rate } \\
(\mathbf{k W})\end{array}$ & $\begin{array}{c}\text { Time to } \\
\text { peak }(\mathbf{m i n})\end{array}$ & $\begin{array}{c}\text { Total energy } \\
\text { released (MJ) }\end{array}$ & $\begin{array}{c}\text { Total mass } \\
\text { loss }(\mathbf{k g})\end{array}$ \\
\hline M1 & 3630 & 12.4 & 3100 & 100 \\
M2 & 3439 & 10.0 & 2100 & 138 \\
M3 & 4063 & 24.1 & 4090 & 184 \\
M4 & 1710 & 27.6 & 3200 & 108 \\
M5 & 4549 & 15.4 & 3466 & 139 \\
M6 & 3560 & 12.0 & 1500 & N/A \\
\hline
\end{tabular}

Table 13 Passenger car : light

\begin{tabular}{lcccc}
\hline Experiments & $\begin{array}{c}\text { Peak heat } \\
\text { release rate } \\
(\mathbf{k W})\end{array}$ & $\begin{array}{c}\text { Time to } \\
\text { peak }(\mathbf{m i n})\end{array}$ & $\begin{array}{c}\text { Total energy } \\
\text { released }(\mathbf{M J})\end{array}$ & $\begin{array}{c}\text { Total mass } \\
\text { loss }(\mathbf{k g})\end{array}$ \\
\hline L1 & 1859 & 24.3 & 3000 & 143 \\
L2 & 1521 & 33.4 & 3300 & 141 \\
L3 & 4470 & 17.0 & 8000 & 270 \\
L4 & 1972 & 12.0 & 3900 & 176 \\
L5 & 8482 & 15.2 & 4008 & $\mathrm{~N} / \mathrm{A}$ \\
L6 & 4390 & 14.4 & 4957 & $\mathrm{~N} / \mathrm{A}$ \\
L7 & 8872 & 20.8 & 4134 & 165 \\
\hline
\end{tabular}

\section{Heat release rate data}

The heat release rate curves presented in this paper are gathered by their classification group Figures 1, 2, 3, 4, 5, 6, 7 and 8 . The unit for heat release rate and the time are standardized to kilowatts $(\mathrm{kW})$ and minutes respectively. So that the reader can easily get a sense of the relative magnitude of the data for each classification, the scales of the plots are fixed corresponding to the maximum of the heat release rate and time. However for heavy passenger cars, the first six plots $(\mathrm{H} 1-6)$ are scaled to $3,500 \mathrm{~kW}$ for heat release rate and $20 \mathrm{~min}$ for the time while the final plot $(\mathrm{H} 7)$ is scaled to $3,500 \mathrm{~kW}$ for heat release rate and $100 \mathrm{~min}$ for the time. Similarly for MPVs, all the plots are fixed at the heat release rate of 6,500 kW however; for the time scale, the first three plots (MPV1 - 3) are scaled to 10 min while the other three plots (MPV4 - 6) are scaled to $70 \mathrm{~min}$.

In experiment MPV3 the fire was extinguished after 4 min and for experiment MED3 it is not mentioned in the source why the fire stopped at 56 min thus making the data incomplete. Experiments H1, H2, H3, H4, H5, H6, SUV1, SUV2, MPV1, MPV2 come from a related set of sources in which were all suppressed after a specific time. These experiments were all representative of postaccident fires carried out for the NHTSA. Experiment M5, L7, H2, H6, MPV4 and U1 have been adjusted to

Table 14 Passenger car : compact

\begin{tabular}{lcccc}
\hline Experiments & $\begin{array}{c}\text { Peak heat } \\
\text { release rate } \\
(\mathbf{k W})\end{array}$ & $\begin{array}{c}\text { Time to } \\
\text { peak (min) }\end{array}$ & $\begin{array}{c}\text { Total energy } \\
\text { released (MJ) }\end{array}$ & $\begin{array}{c}\text { Total mass } \\
\text { loss }(\mathbf{k g})\end{array}$ \\
\hline C1 & 3801 & 24.1 & 5280 & 165 \\
C2 & 8188 & 25.2 & 6670 & 275 \\
C3 & 3560 & 31.0 & 4950 & 225 \\
C4 & 3633 & 25.0 & 4860 & 221 \\
C5 & 1990 & 67.0 & 4930 & 224 \\
C6 & 3039 & 55.0 & 5040 & 229 \\
C7 & 4800 & 37.5 & N/A & N/A \\
\hline
\end{tabular}


Table 15 Passenger car : medium

\begin{tabular}{lcccc}
\hline Experiments & $\begin{array}{c}\text { Peak heat } \\
\text { release rate } \\
(\mathbf{k W})\end{array}$ & $\begin{array}{c}\text { Time to } \\
\text { peak }(\mathbf{m i n})\end{array}$ & $\begin{array}{c}\text { Total energy } \\
\text { released }(\mathbf{M J})\end{array}$ & $\begin{array}{c}\text { Total mass } \\
\text { loss }(\mathbf{k g})\end{array}$ \\
\hline MED1 & 4073 & 38.3 & 6144 & 192 \\
MED2 & 8283 & 36.9 & 7000 & 255 \\
MED3 & 9854 & 37.8 & 6806 & 262 \\
MED4 & 3650 & 46.9 & 5960 & 186 \\
MED5 & 8354 & 26.0 & 6700 & N/A \\
\hline
\end{tabular}

exclude the incipient stage of the fire, which shows no values or values too small to record. The adjusted time for experiments M5, L7, H2, H6, MPV4 and MPV6 were $1 \mathrm{~min} 18 \mathrm{~s} ; 5 \mathrm{~min} ; 17 \mathrm{~min} 47 \mathrm{~s} ; 1 \min 51 \mathrm{~s} ; 7 \mathrm{~min} 42 \mathrm{~s}$; and $3 \mathrm{~min}$ and $6 \mathrm{~s}$ respectively.

A summary of the peak heat release rate, the time to reach the peak heat release rate and the total energy released is given in Tables 12, 13, 14, 15, 16, 17, 18 and 19. If the information is not explicitly stated in the corresponding reference sources then it is obtained from the curves. The total mass loss of the vehicles during the experiment is also stated if it is available from the references otherwise the tables indicates "N/A".

\section{Analysis and discussion}

To perform further analysis of the data collected there are several factors that need to be considered. Firstly it is already noted that there has been a change in the types of materials used in vehicles over the 40-year span of experiments assessed in this work. These changes in materials have been notably in the plastics and composite content which have increased up to $181.4 \mathrm{~kg}$ (400 lbs) per vehicle in 2010s compared to less than $9.1 \mathrm{~kg}$ (20 lbs) per vehicle in the 1960s (Swift 2012). These changes could result in differences in the fire severity characteristics of a vehicle even if the weight is the same due to changing amount and calorific value of the combustible materials. In addition it is also noted that the
Table 17 Sport-utility vehicle (SUV)

\begin{tabular}{lcccc}
\hline Experiments & $\begin{array}{c}\text { Peak heat } \\
\text { release rate } \\
\mathbf{( k W )}\end{array}$ & $\begin{array}{c}\text { Time to } \\
\text { peak (min) }\end{array}$ & $\begin{array}{c}\text { Total energy } \\
\text { released (MJ) }\end{array}$ & $\begin{array}{c}\text { Total mass } \\
\text { loss (kg) }\end{array}$ \\
\hline SUV1 & 484 & 4.3 & 90 & N/A \\
SUV2 & 1337 & 2.5 & 131 & N/A \\
\hline
\end{tabular}

procedures, standards and/or protocols varied between each experiment which likely lead to different effects on the fire spread, availability of air etc. Finally it is important to note that the various heat release rate measurement techniques, namely mass loss rate, convective calorimetry and species-based calorimetry, could result in variability in the heat release rate measurements (Biteau et al. 2008) thus affecting the fire severity analyses.

As already discussed, this work will consider curb weight which is deemed sufficient for the purposes of the wider research objectives associated with a riskbased fire safety of passenger road vehicles in parking buildings. However even this grouping already reduces the data set size to a maximum of seven experiments which challenges any statistical analysis. The factors that include the age of the vehicle, the heat release rate measurement technique, the ignition conditions, the availability of air etc. mean that any analysis that groups the vehicle data together will result in heterogeneous data sets to some degree. It is not possible to create absolutely homogenous data sets that also provide sufficient items of data to be meaningful.

\section{Vehicle age}

Since it is noted that the amount of combustible materials in vehicles has changed over time, a vehicle age analysis for the 41 experiments is carried out. The data collated in this paper includes passenger vehicles manufactured of a span of 40 years however not all of the references provided the exact year of vehicle manufacture thus the experiments were divided into three age categories; experiments with the known year of vehicle

Table 18 Multi-purpose vehicle (MPV)

\begin{tabular}{lcccc}
\hline Experiments & $\begin{array}{c}\text { Peak heat } \\
\text { release rate } \\
(\mathbf{k W})\end{array}$ & $\begin{array}{c}\text { Time to } \\
\text { peak }(\mathbf{m i n})\end{array}$ & $\begin{array}{c}\text { Total energy } \\
\text { released (M) }\end{array}$ & $\begin{array}{c}\text { Total mass } \\
\text { loss }(\mathbf{k g})\end{array}$ \\
\hline $\mathrm{H} 1$ & 780 & 2.6 & 1816 & $\mathrm{~N} / \mathrm{A}$ \\
$\mathrm{H} 2$ & 1189 & 27.1 & 199 & $\mathrm{~N} / \mathrm{A}$ \\
$\mathrm{H} 3$ & 1181 & 3.8 & 130 & $\mathrm{~N} / \mathrm{A}$ \\
$\mathrm{H} 4$ & 2973 & 12.7 & 445 & $\mathrm{~N} / \mathrm{A}$ \\
$\mathrm{H} 5$ & 3173 & 12.9 & 540 & $\mathrm{~N} / \mathrm{A}$ \\
$\mathrm{H} 6$ & 1161 & 16.0 & 233 & $\mathrm{~N} / \mathrm{A}$ \\
$\mathrm{H} 7$ & 3332 & 34.4 & 7648 & 239 \\
\hline
\end{tabular}

\begin{tabular}{lcccc}
\hline Experiments & $\begin{array}{c}\text { Peak heat } \\
\text { release rate } \\
(\mathbf{k W})\end{array}$ & $\begin{array}{c}\text { Time to } \\
\text { peak }(\mathbf{m i n})\end{array}$ & $\begin{array}{c}\text { Total energy } \\
\text { released }(\mathbf{M J})\end{array}$ & $\begin{array}{c}\text { Total mass } \\
\text { loss }(\mathbf{k g})\end{array}$ \\
\hline MPV1 & 4797 & 4.6 & 421 & $\mathrm{~N} / \mathrm{A}$ \\
MPV2 & 1545 & 10.7 & 254 & $\mathrm{~N} / \mathrm{A}$ \\
MPV3 & 2405 & 2.3 & 459 & $\mathrm{~N} / \mathrm{A}$ \\
MPV4 & 4270 & 15.8 & 5028 & 201 \\
MPV5 & 3800 & 54.0 & $\mathrm{~N} / \mathrm{A}$ & $\mathrm{N} / \mathrm{A}$ \\
MPV6 & 6206 & 9.2 & 7000 & $\mathrm{~N} / \mathrm{A}$ \\
\hline
\end{tabular}


Table 19 Unclassified vehicle

\begin{tabular}{lcccc}
\hline Experiments & $\begin{array}{c}\text { Peak heat } \\
\text { release } \\
\text { rate }(\mathbf{k W})\end{array}$ & $\begin{array}{c}\text { Time to } \\
\text { peak (min) }\end{array}$ & $\begin{array}{c}\text { Total energy } \\
\text { released (MJ) }\end{array}$ & $\begin{array}{c}\text { Total mass } \\
\text { loss }(\mathbf{k g})\end{array}$ \\
\hline U1 & 3618 & 28.4 & 3800 & N/A \\
\hline
\end{tabular}

manufacture; experiments with the known decade of vehicle manufacture and experiments with an estimated decade of vehicle manufacture based on the date of experiment, date of submission or publication of the source reference. Clearly the third age category introduces more uncertainty to the year of vehicle manufacture than the other two age categories.

There are 20 experiments that give a known year of manufacture. However, from these 20 experiments there are limitations on the results from the 12 experiments $\mathrm{H} 1$, H2, H3, H4, H5, H6, SUV1, SUV2, MPV1, MPV2, MPV3 and U1 (all manufactured during the 1990s) such that they are not suitable for complete analysis. There are 11 experiments with a known decade of manufacture while the remaining 10 experiments only have an estimated decade of manufacture based on the date of the experiment, and/ or publication. For these 10 cases, five could only be dated somewhere between 1970 and the end of the 1990s, while three could only be dated somewhere between 1980 and the end of the 1990s, and the remaining two could only be dated somewhere between 1960 and the end of the 1990s. Figure 9 shows those dates for the first two age categories described above since data including the third age category gave widely dispersed results. It can be seen that $70 \%$ of the vehicles examined in this study were manufactured during the 1990s.

\section{Fire severity analysis}

Table 20 gathers the results for the 41 experiments for the mean and standard deviation of peak heat release rate $(\mathrm{kW})$, time to reach peak heat release rate (min) and total energy released (MJ) for each classification. Table 20 suggests that the three fire severity characteristics generally increase with curb weight up to the Passenger car : Medium class where it is assumed that the combustible material content can be regarded as likely being reasonably homogeneous given a large majority of vehicles were manufactured during the 1990s. An assessment of these findings is provided later.

However as noted earlier, there are limitations on the results from experiments $\mathrm{H} 1, \mathrm{H} 2, \mathrm{H} 3, \mathrm{H} 4, \mathrm{H} 5, \mathrm{H} 6$, SUV1, SUV2, MPV1, MPV2 and MPV3 as these experiments were suppressed prior to complete vehicle burnout or the data are otherwise incomplete. These limitations affect the usefulness of the statistics for Passenger Car: Heavy, SUV and MPV classifications and as a result, these curb weight classes are predominately excluded from the further analysis as the results would not likely represent the behaviour of vehicle fires for its class. Thus Table 20 also provides an analysis that excludes the 11 experiments listed above along with experiment $\mathrm{U} 1$ due to the lack of details.

Graphs of the total energy released, the time to reach the peak heat release rate and peak heat release rate are constructed for the four classifications with sufficient complete data, namely: Passenger Car: Mini, Light, Compact and Medium data sets. Additionally, four data points; three from MPV4, MPV5 and MPV6 and one from Passenger Car: Heavy (H7) are also included in the graphs. For experiments M5, M7, L5, L7 and MED5, horizontal bars are shown to represent the range of possible vehicle curb weights.

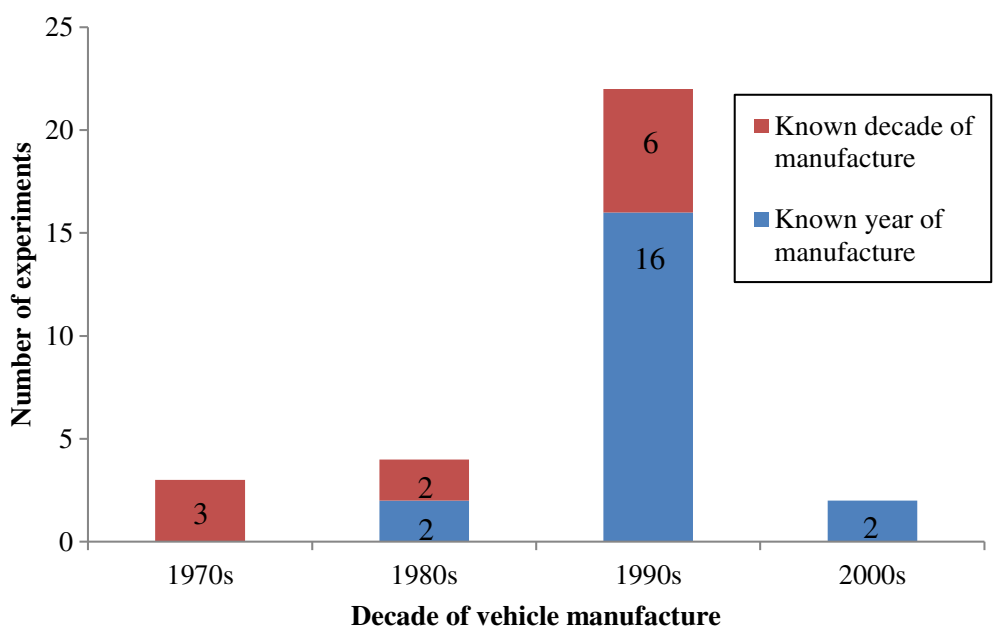

Figure 9 The experiments' vehicle age distribution over four decades. 
Table 20 Mean and standard deviation fire severity characteristics for all experiments by curb weight classification

\begin{tabular}{|c|c|c|c|c|c|c|c|c|c|c|c|c|}
\hline \multirow{3}{*}{$\begin{array}{l}\text { Vehicle } \\
\text { classification }\end{array}$} & \multicolumn{4}{|c|}{ Peak heat release rate } & \multicolumn{4}{|c|}{ Time to peak } & \multicolumn{4}{|c|}{ Total energy released } \\
\hline & \multicolumn{4}{|c|}{$(\mathrm{kW})$} & \multicolumn{4}{|c|}{$(\min )$} & \multicolumn{4}{|c|}{ (MJ) } \\
\hline & Mean & $\begin{array}{l}\text { Standard } \\
\text { deviation }\end{array}$ & $\begin{array}{l}\text { Max } \\
\text { value }\end{array}$ & $\begin{array}{l}\text { Min } \\
\text { value }\end{array}$ & Mean & $\begin{array}{l}\text { Standard } \\
\text { deviation }\end{array}$ & $\begin{array}{l}\text { Max } \\
\text { value }\end{array}$ & $\begin{array}{l}\text { Min } \\
\text { value }\end{array}$ & Mean & $\begin{array}{l}\text { Standard } \\
\text { deviation }\end{array}$ & $\begin{array}{l}\text { Max } \\
\text { value }\end{array}$ & $\begin{array}{l}\text { Min } \\
\text { value }\end{array}$ \\
\hline $\begin{array}{l}\text { Passenger car: } \\
\text { Mini }\end{array}$ & 3492 & 964 & 4063 & 1710 & 16.9 & 7.2 & 27.6 & 10.0 & 2909 & 945 & 4090 & 1500 \\
\hline $\begin{array}{l}\text { Passenger car: } \\
\text { Light }\end{array}$ & 4509 & 3088 & 8872 & 1521 & 19.6 & 7.4 & 33.4 & 12.0 & 4471 & 1677 & 8000 & 3000 \\
\hline $\begin{array}{l}\text { Passenger car: } \\
\text { Compact }\end{array}$ & 4144 & 1973 & 8188 & 1990 & 37.8 & 16.9 & 67.0 & 25.0 & 5288 & 692 & 6670 & 4860 \\
\hline $\begin{array}{l}\text { Passenger car: } \\
\text { Medium }\end{array}$ & 6843 & 2797 & 9854 & 3650 & 37.2 & 7.4 & 46.9 & 26.0 & 6386 & 695 & 7000 & 5960 \\
\hline Passenger car: & 1969 & 1126 & 3332 & 780 & 15.6 & 11.6 & 34.4 & 2.6 & 1573 & 2740 & 7648 & 130 \\
\hline Heavy & (3332) & $(-)$ & $(-)$ & $(-)$ & $(34.4)$ & $(-)$ & $(-)$ & $(-)$ & $(7648)$ & $(-)$ & $(-)$ & $(-)$ \\
\hline \multirow[t]{2}{*}{ SUV } & 910 & 603 & 1337 & 484 & 3.4 & 1.2 & 4.3 & 2.5 & 110 & 28 & 131 & 90 \\
\hline & $(-)$ & $(-)$ & $(-)$ & $(-)$ & $(-)$ & $(-)$ & $(-)$ & $(-)$ & $(-)$ & $(-)$ & $(-)$ & $(-)$ \\
\hline \multirow[t]{2}{*}{ MPV } & 3837 & 1675 & 6206 & 1545 & 16.1 & 19.2 & 54.0 & 2.3 & 2632 & 3166 & 7000 & 254 \\
\hline & (4759) & (1041) & (6206) & (3800) & $(26.3)$ & (19.7) & $(54.0)$ & $(9.2)$ & (6014) & (986) & (7000) & (5028) \\
\hline \multirow{2}{*}{$\begin{array}{l}\text { Unclassified } \\
\text { vehicle }\end{array}$} & 3618 & - & - & - & 28.4 & - & - & - & 3800 & - & - & - \\
\hline & $(-)$ & $(-)$ & $(-)$ & $(-)$ & $(-)$ & $(-)$ & $(-)$ & $(-)$ & $(-)$ & $(-)$ & $(-)$ & $(-)$ \\
\hline
\end{tabular}

not applicable.

Values shown enclosed in braces do not include those experiments that have been excluded from further analysis.

Figure 10 shows there is a proportional increase in total energy released with the increment of curb weight where the average total energy released increases from around 3500 to $6800 \mathrm{MJ}$. A linear trend through the origin is fitted to the data with a relatively weak $R^{2}$ correlation value of 0.55 . It is noted that the result from L3 appears as an outlier when compared to the remaining data and if this is excluded then the $R^{2}$ value increases to 0.72 . The total energy release over the experiments considered can be approximated as 4.14 times the curb weight whether L3 is included or not. In comparison the vehicle mass categories proposed by (Joyeux et al. 2002) and the linear fit proposed by (Shintani et al. 2004) are shown in Figure 10. It is clear that Joyeux et al.'s values

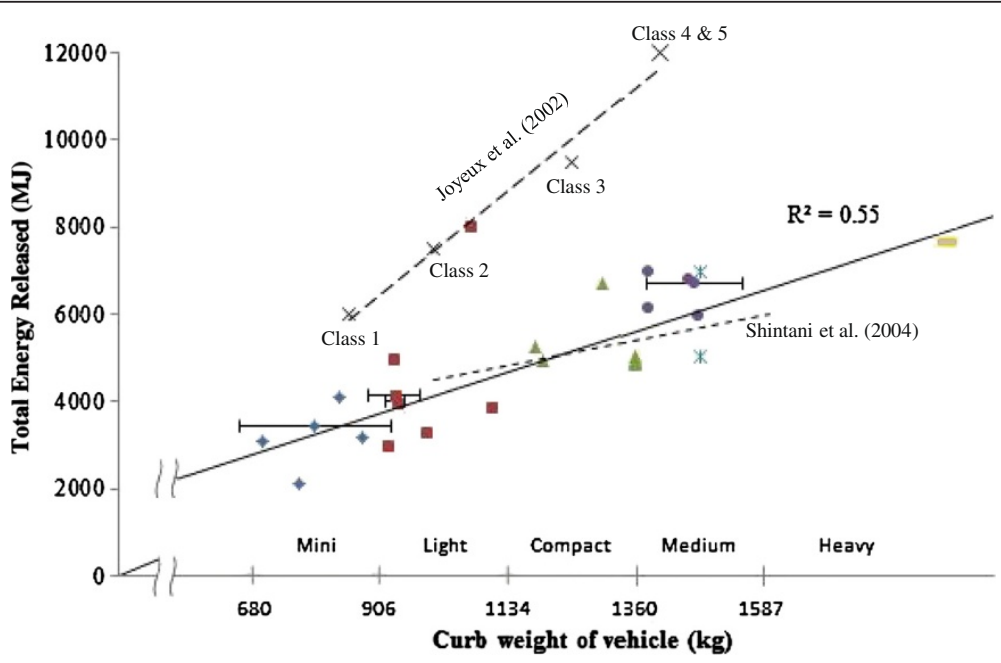

Figure 10 The total energy released against curb weight of vehicles and associated classifications. (Solid symbols correspond to ANSI vehicle curb weight classifications; * symbol for ANSI MPV classification; and × symbol for Joyeux's European Car classification, 1 - 5). 


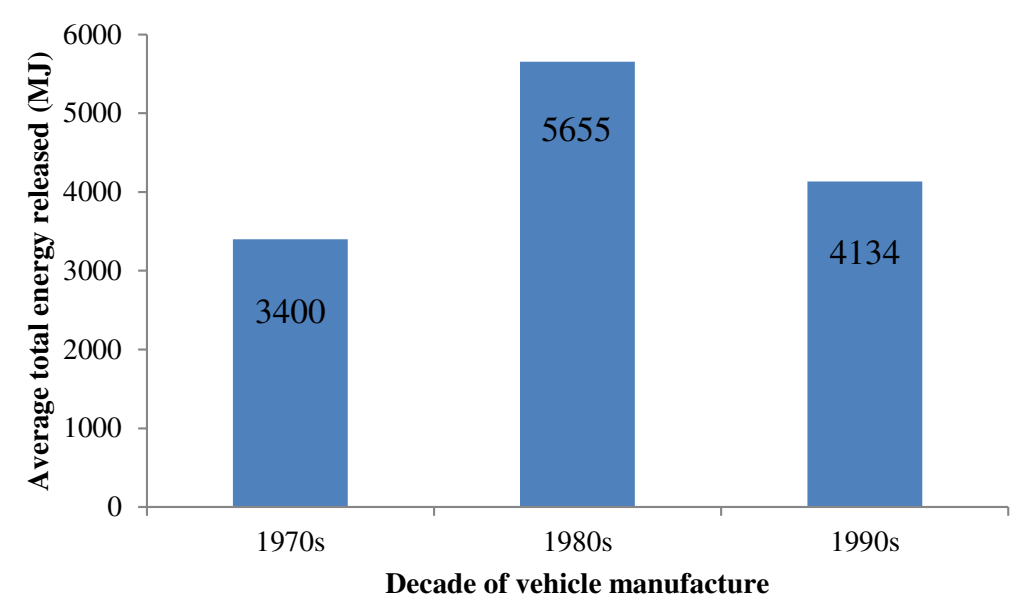

Figure 11 Total energy release for the passenger Car: light curb weight classification over the best estimate of the decade.

for total energy release are noticeably higher for a given vehicle mass as compared to the experimental data with only the outlier from L3 being comparable. Shintani et al.'s linear fit (where their four experiments are included in this analysis as experiments C1, MED1, MED4 and H7) is similar to the proposed linear fit across the majority of the data but an extrapolation to lower curb weights would give disproportionately lower energy release values.

It is useful to use the data to investigate whether the total energy released for a given vehicle category decreases as the vehicle age increases because of the changes in combustible materials over time. However since the majority of vehicles identified in this study are grouped in the 1990s age category and only the
Passenger Car: Light curb weight classification spans more than two decades, it is not possible to get much in the way of firm conclusions on this issue. Figure 11 shows a graph of the average total energy release for the Passenger Car: Light curb weight classification for the best estimate of the decade of vehicle manufacture. Data for the 1980s consists of L3, L5 and L6 where the highest energy release is from L3 at 8000 MJ which is more than $60 \%$ greater than any other value recorded for this curb weight classification. If L3 is treated as an outlier, as previously, then the average total energy release reduces to $4483 \mathrm{MJ}$ which still exceeds the 1990s result from the single L7 experiment. Using the information given by (Swift 2012) for the increase in the amount of plastics per decade, then a vehicle manufactured in

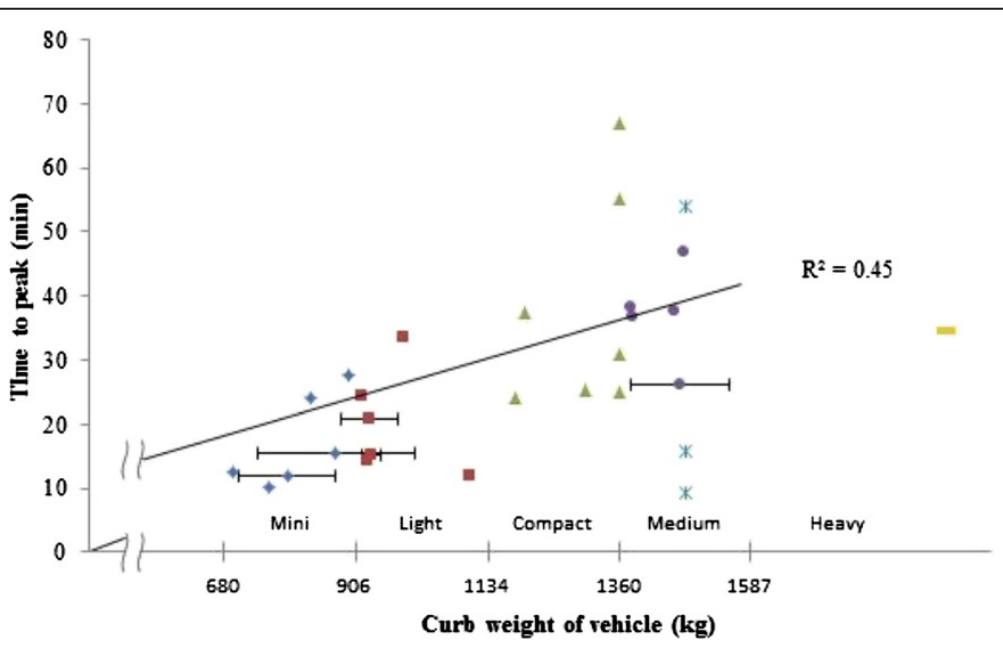

Figure 12 The time to reach peak heat release rate against curb weight of vehicles and associated classifications. (Solid symbols correspond to ANSI vehicle curb weight classifications; and * symbol for ANSI MPV classification). 


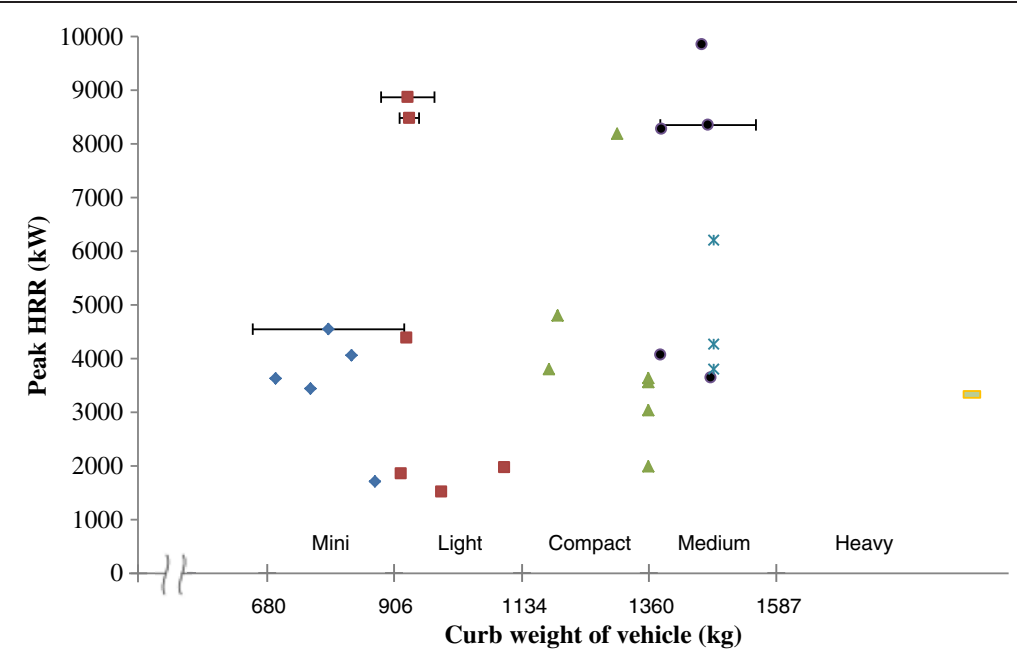

Figure 13 The peak heat release rates against curb weight of vehicles and associated classifications. (Solid symbols correspond to ANSI vehicle curb weight classifications; and * symbol for ANSI MPV classification).

the late 1970s would have around $150 \mathrm{~kg}$ and one manufactured in the 1990s would have around $200 \mathrm{~kg}$, i.e. an increase of $50 \mathrm{~kg}$. Heats of combustion for most typical thermoplastic and thermosetting polymers are in the range $\sim 16-46 \mathrm{MJ} / \mathrm{kg}$ (Drysdale, 2011) and if it is then assumed that only the increase in plastics contributes to the change in the total energy release then this would result in an increase in the range $800-2300 \mathrm{MJ}$. From Figure 11 the change in total energy release is from an average value of $3400 \mathrm{MJ}$ (L1, L2 and L4) expected value would be $4200-5700 \mathrm{MJ}$ compared to around 4134 MJ (for experiment L7) which is less than the lower estimated bound.

The time to reach peak heat release rate in Figure 12 shows a generally increasing trend as the curb weight class of the vehicles increases. A linear trend is fitted to the data up to the Passenger Car: Medium class due to limited adequate data sets for greater curb weights. However the fit only achieves an $\mathrm{R}^{2}$ value of 0.45 with scatter in the data increasing as the curb weight increases. The peak heat release rate in Figure 13 exhibits a very weak correlation with curb weight such that there is a reduction for the Passenger car: Compact class when compared to the preceding lighter class. Although other classes show an increasing trend against the increase of curb weight there is noticeable scatter in the data and so there is no attempt to fit a trend line.

\section{Distribution analysis}

Given the somewhat weak correlation for the linear fits to the total rate of heat release and time to peak rate of heat release it is worthwhile to further examine the data using statistical distributions. For this distribution analysis the curb weight classes Passenger car: Mini, Light, Compact and Medium are investigated as these groups had sufficient data available. In addition, a distribution that combines the passenger vehicles for these four curb weight classes is obtained in order to utilize a larger data set and provide a more generic distribution that encompasses the four classifications.

There are three methods used by the @RISK software for obtaining the best-fit probability distributions: the

Table 21 Ranked order distributions for peak heat released rate for combined vehicles

\begin{tabular}{|c|c|c|c|c|c|c|c|c|}
\hline \multirow[b]{2}{*}{ Rank } & \multicolumn{2}{|l|}{ Mini } & \multicolumn{2}{|l|}{ Light } & \multicolumn{2}{|l|}{ Compact } & \multicolumn{2}{|l|}{ Medium } \\
\hline & Distribution shape & Value & Distribution shape & Value & Distribution shape & Value & Distribution shape & Value \\
\hline 1 & Weibull & 0.28 & Log-Logistic & 0.24 & Lognormal & 0.22 & Beta General & 0.30 \\
\hline 2 & Beta General & 0.29 & Weibull & 0.24 & Gamma & 0.24 & Triangular & 0.31 \\
\hline 3 & Gamma & 0.35 & Triangular & 0.25 & Weibull & 0.26 & Weibull & 0.32 \\
\hline 4 & Lognormal & 0.37 & Lognormal & 0.25 & Triangular & 0.28 & Gamma & 0.34 \\
\hline 5 & Triangular & 0.41 & Gamma & 0.25 & Beta General & 0.35 & Lognormal & 0.34 \\
\hline
\end{tabular}


Table 22 Ranked order distributions for time to reach peak heat release rate for combined vehicles

\begin{tabular}{|c|c|c|c|c|c|c|c|c|}
\hline \multirow[b]{2}{*}{ Rank } & \multicolumn{2}{|l|}{ Mini } & \multicolumn{2}{|l|}{ Light } & \multicolumn{2}{|l|}{ Compact } & \multicolumn{2}{|l|}{ Medium } \\
\hline & Distribution shape & Value & Distribution shape & Value & Distribution shape & Value & Distribution shape & Value \\
\hline 1 & Log-Logistic & 0.21 & Log-Logistic & 0.15 & Log-Logistic & 0.21 & Weibull & 0.27 \\
\hline 2 & Lognormal & 0.24 & Lognormal & 0.18 & Weibull & 0.22 & Gamma & 0.31 \\
\hline 3 & Weibull & 0.24 & Gamma & 0.19 & Gamma & 0.23 & Lognormal & 0.32 \\
\hline 4 & Gamma & 0.25 & Weibull & 0.20 & Lognormal & 0.24 & Triangular & 0.42 \\
\hline 5 & Beta General & 0.27 & Triangular & 0.24 & Triangular & 0.29 & & \\
\hline
\end{tabular}

Table 23 Ranked order distributions for total energy released for combined vehicles

\begin{tabular}{|c|c|c|c|c|c|c|c|c|}
\hline \multirow[b]{2}{*}{ Rank } & \multicolumn{2}{|l|}{ Mini } & \multicolumn{2}{|l|}{ Light } & \multicolumn{2}{|l|}{ Compact } & \multicolumn{2}{|l|}{ Medium } \\
\hline & Distribution shape & Value & Distribution shape & Value & Distribution shape & Value & Distribution shape & Value \\
\hline 1 & Triangular & 0.21 & Triangular & 0.25 & Lognormal & 0.32 & Beta General & 0.25 \\
\hline 2 & Weibull & 0.24 & Lognormal & 0.25 & Gamma & 0.32 & Weibull & 0.27 \\
\hline 3 & Gamma & 0.29 & Gamma & 0.27 & Weibull & 0.36 & Gamma & 0.30 \\
\hline 4 & Lognormal & 0.30 & Weibull & 0.28 & Beta General & 0.45 & Lognormal & 0.30 \\
\hline 5 & Beta General & 0.32 & Beta General & 0.45 & Triangular & 0.53 & Triangular & 0.57 \\
\hline
\end{tabular}

Chi-squared method, the Anderson-Darling method and the Kolmogorov-Smirnov method. For this particular analysis, the Kolmogorov-Smirnov method was chosen as this method is focused on the middle of the distribution. The outcome of the @RISK distribution fitting process is a ranked order of fitting statistics for each potential distribution shape where a smaller value indicates a better fit. Nevertheless, for this particular analysis, the selections of distribution shapes were not only based on the ranking of the fitting statistics values but also based on the distribution shapes that are commonly used and likely to be available in other software tools for further analysis, and also on selecting a consistent distribution shape for the various severity characteristics. For the fire risk analysis in parking building research the B-RISK model (Wade et al. 2013) is planned to be used for the analysis of fire spread between vehicles and so distribution shapes that have the potential to be used in this model have been selected. The distributions selected for the ranking in @RISK are Weibull, Beta General, Gamma, Lognormal, Log-Logistic and Triangular all with the lower bound fixed at zero where relevant.
Tables 21, 22 and 23 shows the ranked order of distributions given by @RISK along with the associated fitting statistic. From this ranking it is decided that the Weibull distribution gives an acceptable overall result. It is clear that in some cases there is very little to choose between the fitting statistics for a given severity characteristic, for example although the Weibull distribution for the time to peak rate of heat release for Passenger car: Mini is the $3^{\text {rd }}$ ranked in the list, the fitting statistic only decrease from 0.21 to 0.24 between the top ranked Log-Logistic and the Weibull distributions. Furthermore although the Weibull distribution is ranked $4^{\text {th }}$ for the time to peak rate of heat release for Passenger car: Light the fitting statistic is still higher than the top-ranked distribution for the four other curb weight classifications.

Table 24 shows the summary of the distribution analysis for four vehicle classifications and all vehicles with the suggested distribution statistics for peak heat release rate, time to reach peak heat release rate and total energy released. Figure 14 shows the frequency data and best-fit distributions for peak heat release rate, time to reach peak

Table 24 Summary of the distribution analyses

\begin{tabular}{|c|c|c|c|c|c|c|c|}
\hline & \multirow[b]{2}{*}{ Distribution parameter } & \multicolumn{2}{|c|}{ Peak heat release rate $(\mathrm{kW})$} & \multicolumn{2}{|c|}{ Time to peak (min) } & \multicolumn{2}{|c|}{ Total energy released (MJ) } \\
\hline & & $a$ & $\beta$ & $a$ & $\beta$ & $a$ & $\beta$ \\
\hline \multirow{5}{*}{ Curb weight class } & Mini & 5.19 & 3809 & 2.79 & 19.1 & 4.02 & 3222 \\
\hline & Light & 1.66 & 5078 & 3.03 & 22.0 & 2.93 & 5009 \\
\hline & Compact & 2.40 & 4691 & 2.60 & 42.8 & 7.49 & 5591 \\
\hline & Medium & 3.18 & 7688 & 6.55 & 39.9 & 14.53 & 6648 \\
\hline & Combined & 2.03 & 5256 & 2.12 & 31.3 & 3.23 & 5233 \\
\hline
\end{tabular}



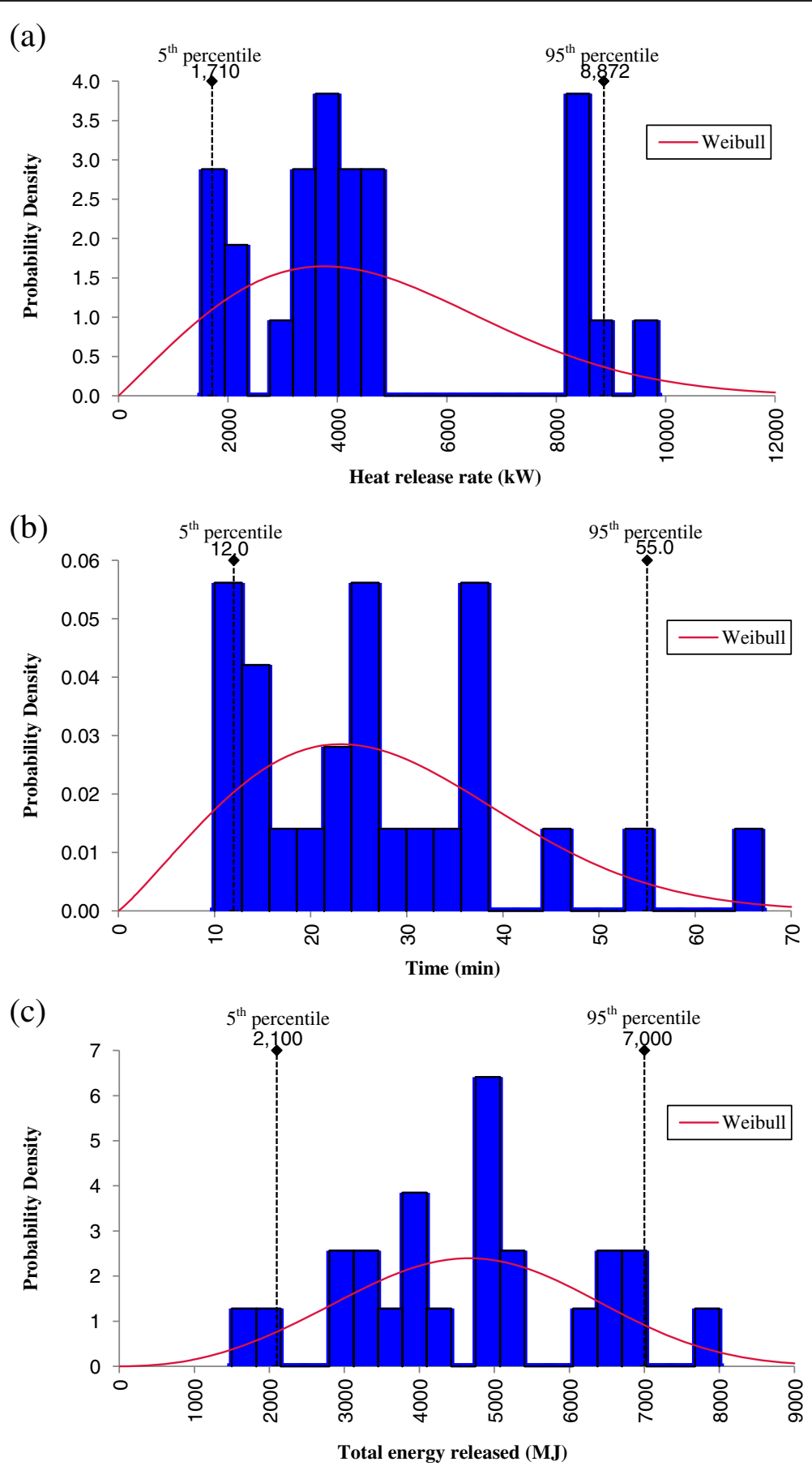

Figure 14 All passenger vehicle frequency data and best-fit distributions: (a) peak heat release rate, (b) of time to peak heat release rate, (c) total energy released.

heat release rate and total energy released for the combined passenger vehicle data. The $5^{\text {th }}$ and $95^{\text {th }}$ percentile values for each distribution are also indicated in Figure 14.

\section{Conclusions and recommendations}

Experimental data for 41 single passenger vehicles have been obtained from the literature. Grouping these experiments by the curb weight of the vehicles forms a useful classification system that can be related to vehicle population and severity where the severity is defined here as the peak heat release rate, the time to reach peak heat release rate and total energy released.

For curb weight classes up to Passenger car : Medium it is found that the average values for the three fire 
severity characteristics generally increase as the curb weight increases. Previous studies have suggested there is a linear the increase in total energy released with curb weight. This study has also obtained a linear fit albeit with a relatively weak correlation. Similarly for the time to reach peak heat release rate a trend is also replicated in the plot of individual data. However, plotting individual results for the peak heat release rate do not clearly exhibit a strong trend that is suggested by the average values because of the scatter in the data.

The literature has found that the amount of combustible materials such as plastics in vehicles has increased since the 1960s. Although the age of the vehicles assessed in this review spans around four decades, it is found that it is sometimes difficult to even ascertain the decade in which an individual vehicle had been manufactured. Of those vehicles for which the decade could be determined with reasonable confidence it is found that around $70 \%$ were manufactured in the $1990 \mathrm{~s}$ and data that spans multiple decades is not generally available for each curb weight class. As a result it is not possible to fully investigate the impact of vehicle age on the fire severity characteristics and thus the findings presented in this review should be treated with some care.

Weibull distribution functions have been obtained for the curb weights up to the Passenger car : Medium class and the combination of these classes. These distributions can be used to assess single-vehicle peak heat release rate, time to reach peak heat release rate and total energy released in a probabilistic manner which can aid designers wishing to perform probabilistic assessment analysis for cost-risk-optimized fire protection design.

It is recommended that the heat release rate for single passenger vehicles is examined again in the future to account for changes in vehicle design, construction and use. Technological advancements will likely include changes in materials used in which could affect the fire behaviour of vehicles. All of the vehicles examined in this review are either petrol (gasoline) or diesel fuelled. For future experiments, it is recommended that research be conducted on vehicles using alternative fuels such as liquefied petroleum gas (LPG), hydrogen, electric power and solar power.

\section{Abbreviations}

NZTA: New Zealand transport agency; ANSI: American national standard; EU: European union; LPG: Liquefied petroleum gas.

\section{Competing interests}

Both authors declare that they have no competing interests.

\section{Authors' contributions}

Both authors discussed the concept of the paper. MZMT collected all the data, performed the analysis and draft the paper. MS edited and revised the manuscript. Both authors read and approved the final manuscript.
Received: 23 April 2013 Accepted: 13 September 2013

Published: 24 September 2013

\section{References}

(2012) NZ transport agency., http://www.nzta.govt.nz/vehicle/classes-standards/ class.html. Accessed 3 December 2012

(2013a) Automobile and Car specifications., http://www.carfolio.com. Accessed 20 February 2013

(2013b) Automotive technical specifications., http://www.carspector.com. Accessed 20 February 2013

(2013c) Car technical specifications., http://www.autofiles.org. Accessed 20 February 2013

American National Standard (2007) Manual on classification of motor vehicle traffic accidents. ANSI D16:1-2007

Babrauskas V, Peacock RD (1992) Heat release rate: the single most important variable in fire hazard. Fire Saf J 18(3):255-272

Biteau H, Steinhaus T, Schemel C, Simeoni A, Marlair G, Bal N, Torero JL (2008) Calculation methods for the heat release rate of materials of unknown composition. Fire Saf Sc 9:1165-1176. doi:10.3801/IAFSS.FSS.9-1165

Carvel RO (ed) (2004) Fire size in tunnels, PhD thesis. Division of Civil Engineering, Heriot-Watt University, Scotland

Cheong MK, Spearpoint MJ, Fleischmann CM (2008) Using peak heat release rate to determine the fire risk level of road tunnels. J Risk and Reliability 222 (4):95-604

Department for Communities and Local Government (CLG) (2010) Fire spread in Car parks BD2552 final report. Department for Communities and Local Government. UK, London

Drysdale D (2011) Introduction to fire dynamics, 3rd edn. John Wiley \& Sons, New York

European Union (2012) Eurostat: regional transport statistics., http://epp.eurostat ec.europa.eu/portal/page/portal/transport/data/database. Accessed 5 December 2012

Fleischmann CM (2008) Proposed framework for performance based fire engineering design in the next generation New Zealand building code: specification of the design fire. SFPE conference on performance based codes and fire safety. SFPE Conference on Performance Based Codes and Fire Safety Design, Auckland

Ingason H (2006) Design fires in tunnels, Safe and reliable tunnels, innovative European achievements, second international symposium. , Lausanne, Switzerland, http://www.unitracc.com/aktuelles/kalender/2nd-internationalsymposium-safe-reliable-tunnels-en

Janssens M (2008) Development of a database of full-scale calorimeter tests of motor vehicle burns. Fire Technology Department, Southwest Research Institute, San Antonio, Texas, USA, http://www.swri.org/

Joyeux D (1997) Natural fires in closed Car parks - Car fire tests, CTICM report No. INC 96/294d DJ/NB. CTICM, Metz, France

Joyeux D, Kruppa J, Cajot L, Schleich J, Van de Leur P, Twilt L (2002) Demonstration of real fire tests in Car parks and high buildings, Final report, contract No. 7215 PP 025. CTICM, Metz, France

Lönnermark A, Blomqvist $P$ (2006) Emissions from an automobile fire. Chemosphere 62:1043-1056

Mangs J, Keski-Rahkonen O (1994) Characterization of the fire behavior of a burning passenger Car. Part I: Car fire experiments. Fire Saf J 23:17-35

Marlair G, Lemaire T, Ohlin M (2008) Fire scenarios and accidents in the past, Workpackage 2 fire development and mitigation measure D211, UPTUN. European Commission under the UPTUN project

Okamoto K, Watanabe N, Hagimoto Y, Chigira T, Masano R, Miura H, Ochiai S, Satoh H, Tamura Y, Hayano K, Maeda Y, Suzuki J (2009) Burning behavior of sedan passenger cars. Fire Saf J 44:301-310

Opland L (2007) Size classification of passenger cars. Master's Thesis, Chalmers University of Technology, Göteborg, Sweden

Palisade Corporation (2010) Guide to using @RISK version 5.7. Palisade Corporation, Ithaca, NY USA

Santrock J (2001) Evaluation of motor vehicle fire initiation and propagation, part 3: propagation in an engine compartment fire in a 1996 passenger Van, NHTSA 1998 3588-119. General Motors Corporation, Warren, MI

Santrock J (2002a) Demonstration of enhanced fire safety technology-fire retardant materials-part 1, Full scale vehicle fire tests of a control vehicle and a test vehicle containing an HVAC module made from polymers containing flame retardant chemicals. NHTSA 1998-3588-190. General Motors Corporation, Warren, MI 
Santrock J (2002b) Evaluation of motor vehicle fire initiation and propagation, part 10: propagation of a Mid-underbody gasoline pool fire in a 1998 sport utility vehicle, NHTSA 1998-3588-189. General Motors Corporation, Warren, MI

Santrock J (2002c) Evaluation of motor vehicle fire initiation and propagation, part 4: propagation of an underbody gasoline pool fire in a 1996 passenger van, NHTSA 1998 3588-143. General Motors Corporation, Warren, Ml

Santrock J (2002d) Evaluation of motor vehicle fire initiation and propagation, part 6: propagation of an underbody gasoline pool fire in a 1997 rear wheel drive passenger car, NHTSA 1998-3588-158. General Motors Corporation, Warren, Ml

Santrock J (2002e) Evaluation of motor vehicle fire initiation and propagation, part 7: propagation of an engine compartment fire in a 1997 rear wheel drive passenger car, NHTSA 1998-3588-178. General Motors Corporation, Warren, MI

Santrock J (2002f) Evaluation of motor vehicle fire initiation and propagation, part 9: propagation of a rear-underbody gasoline pool fire in a 1998 sport utility vehicle, NHTSA 1998-3588-188. General Motors Corporation, Warren, MI

Santrock J (2003a) Evaluation of motor vehicle fire initiation and propagation, part 12: propagation of an underbody gasoline pool fire in a 1998 frontwheel drive passenger vehicle, NHTSA 1998-3588-201. General Motors Corporation, Warren, Ml

Santrock J (2003b) Evaluation of motor vehicle fire initiation and propagation, part 13: propagation of an engine compartment fire in a 1998 front-wheel drive passenger vehicle, NHTSA 1998-3588-203. General Motors Corporation, Warren, MI

Shintani Y, Kakae N, Harada K, Masuda H, Takahashi W (2004) Experimental investigation of burning behavior of automobiles, 6th Asia-Oceania symposium on fire science and technology. IAFSS (International Association for Fire Safety Science), Secretariat: Interscience Communications Ltd, West Yard House, Guildford Grove, London, UK, pp 618-629

Shipp M, Spearpoint M (1995) Measurements of the severity of fires involving private motor vehicles. Fire Mater 19:143-151

Steinert C (1994) Smoke and heat production in tunnel fires, International conference on fires in tunnels, 123-137. Swedish National Testing and Research Institute, Borås, Sweden

Steinert C (2000) Experimental investigation of burning and fire jumping behavior of automobiles (in German). VFDB 49:163-172

Stroup D, DeLauter L, Lee L, Roadermel G (2001) Passenger minivan fire tests, FR 4011. National Institute of Standards and Technology, Gaithersburg, MD

Swift K (2012) Chemistry and light vehicle annual report. Economics \& Statistics Department, American Chemistry Council, Washington, DC, USA, http://www. plastics-car.com/

Van Oerle N, Lemaire A, Van de Leur P (1999) Effectiviteit van stuwkrachtventilatie in gesloten parkeergarages, TNO Report No. 1999-CVB-RR1442. TNO, Delft, The Netherlands

Wade C, Baker G, Frank K, Robbins A, Harrison R, Spearpoint M, Fleischmann CM (2013) B-RISK user guide and technical manual, SR 282. BRANZ Ltd, New Zealand

doi:10.1186/2193-0414-2-5

Cite this article as: Mohd Tohir and Spearpoint: Distribution analysis of the fire severity characteristics of single passenger road vehicles using heat release rate data. Fire Science Reviews 2013 2:5.

\section{Submit your manuscript to a SpringerOpen ${ }^{\circ}$ journal and benefit from:}

- Convenient online submission

- Rigorous peer review

- Immediate publication on acceptance

- Open access: articles freely available online

- High visibility within the field

- Retaining the copyright to your article

Submit your next manuscript at $>$ springeropen.com 